\title{
ROBOTIC LASER ADAPTIVE OPTICS IMAGING OF 715 KEPLER EXOPLANET CANDIDATES USING ROBO-AO
}

\author{
Nicholas M. Law ${ }^{1}$, Tim Morton ${ }^{2}$, Christoph Baranec ${ }^{3}$, Reed Riddle $^{2}$, Ganesh Ravichandran $^{4}$, \\ Carl Ziegler $^{1}$, John Asher Johnson ${ }^{5}$, Shriharsh P. Tendulkar ${ }^{2}$, Khanh Bui $^{2}$, Mahesh P. Burse ${ }^{6}$, \\ H. K. Das ${ }^{6}$, Richard G. Dekany ${ }^{2}$, Shrinivas Kulkarni ${ }^{2}$, Sujit Punnadi ${ }^{2}$, and A. N. Ramaprakash ${ }^{6}$ \\ ${ }^{1}$ Department of Physics and Astronomy, University of North Carolina at Chapel Hill, Chapel Hill, NC 27599-3255, USA \\ 2 Division of Physics, Mathematics, and Astronomy, California Institute of Technology, Pasadena, CA 91125, USA \\ ${ }^{3}$ Institute for Astronomy, University of Hawai'i at Mānoa, Hilo, HI 96720-2700, USA \\ ${ }^{4}$ West Tresper Clarke High School, East Meadow School District, 740 Edgewood Drive, Westbury, NY 11590, USA \\ ${ }^{5}$ Harvard-Smithsonian Center for Astrophysics, 60 Garden Street, Cambridge, MA 02138, USA \\ ${ }^{6}$ Inter-University Centre for Astronomy and Astrophysics, Ganeshkhind, Pune 411007, India \\ Received 2013 December 17; accepted 2014 June 2; published 2014 July 24
}

\begin{abstract}
The Robo-AO Kepler Planetary Candidate Survey is observing every Kepler planet candidate host star with laser adaptive optics imaging to search for blended nearby stars, which may be physically associated companions and/ or responsible for transit false positives. In this paper, we present the results from the 2012 observing season, searching for stars close to 715 Kepler planet candidate hosts. We find 53 companions, 43 of which are new discoveries. We detail the Robo-AO survey data reduction methods including a method of using the large ensemble of target observations as mutual point-spread-function references, along with a new automated companion-detection algorithm designed for large adaptive optics surveys. Our survey is sensitive to objects from $\approx 0$ "' 15 to 2".5 separation, with magnitude differences up to $\Delta m \approx 6$. We measure an overall nearby-star probability for Kepler planet candidates of $7.4 \% \pm 1.0 \%$, and calculate the effects of each detected nearby star on the Kepler-measured planetary radius. We discuss several Kepler Objects of Interest (KOIs) of particular interest, including KOI-191 and KOI-1151, which are both multi-planet systems with detected stellar companions whose unusual planetary system architecture might be best explained if they are "coincident multiple" systems, with several transiting planets shared between the two stars. Finally, we find $98 \%$ confidence evidence that short-period giant planets are two to three times more likely than longer-period planets to be found in wide stellar binaries.
\end{abstract}

Key words: binaries: close - instrumentation: adaptive optics - instrumentation: high angular resolution methods: data analysis - methods: observational - planetary systems - planets and satellites: detection planets and satellites: fundamental parameters

Online-only material: color figures

\section{INTRODUCTION}

The Kepler mission, which has searched approximately 190,000 stars for the tiny periodic dips in stellar brightness indicative of transiting planets, is unprecedented in both sensitivity and scale among transiting planet surveys (Koch et al. 2010). Never before has a survey been able to detect such small planets-down to even the size of Earth's moon (Barclay et al. 2013) — and never before has a survey delivered so many planet candidates, with over 3500 planet candidates (candidate Kepler Objects of Interest; KOIs) found in a search of the first 12 quarters of Kepler photometry (Borucki et al. 2010, 2011; Batalha et al. 2013; Tenenbaum et al. 2013).

All exoplanet transit surveys require follow-up observations of the detected candidates. The purpose of this follow-up is twofold: first, to confirm that the detected photometric dimmings are in fact truly transiting planets rather than astrophysical false positives; and second, to characterize the host stellar system. High-angular-resolution imaging is a crucial ingredient of the follow-up effort, as many astrophysical false-positive scenarios involve nearby stellar systems whose light is blended with the target star (e.g., O'Donovan et al. 2006). Even if a transit candidate is a true planet, identifying whether it is in a binary stellar system has potentially important implications for determining the planet's detailed properties. For example, if there is considerable diluting flux from a companion star within the photometric aperture, even if the planet interpretation of the signal is secure, the planet will be larger than implied by the light curve alone under the assumption of a single host star (e.g., Johnson et al. 2011). The presence or absence of third bodies in the systems can also have broader implications about the processes of planetary system formation and evolution; stellar binarity has been hypothesized to be important in shaping the architectures of planetary systems, both by regulating planet formation and by dynamically sculpting planets final orbits, such as forcing Kozai oscillations that cause planet migration (e.g., Fabrycky \& Tremaine 2007; Katz et al. 2011; Naoz et al. 2012) or tilting the circumstellar disk (Batygin 2012).

The vast majority of the individual Kepler candidates remain unconfirmed $(<3 \%$ currently confirmed according to the NASA Exoplanet Archive, NEA). Current predictions based on models of the expected population of confusion sources suggest that at least $10 \%-15 \%$ of Kepler's planetary candidates may be astrophysical false positives and that a large fraction of confirmed planets also have incorrectly determined planetary parameters because of confusing sources (Morton \& Johnson 2011; Fressin et al. 2013; Dressing \& Charbonneau 2013; Santerne et al. 2013). The possible false-positive scenario probabilities change with the brightness of the Kepler target, the details of its Kepler light curve, its spectral type, and the properties of the detected planetary system (e.g., Morton 2012). The false positives thus limit our ability to interpret individual objects, to evaluate differences 

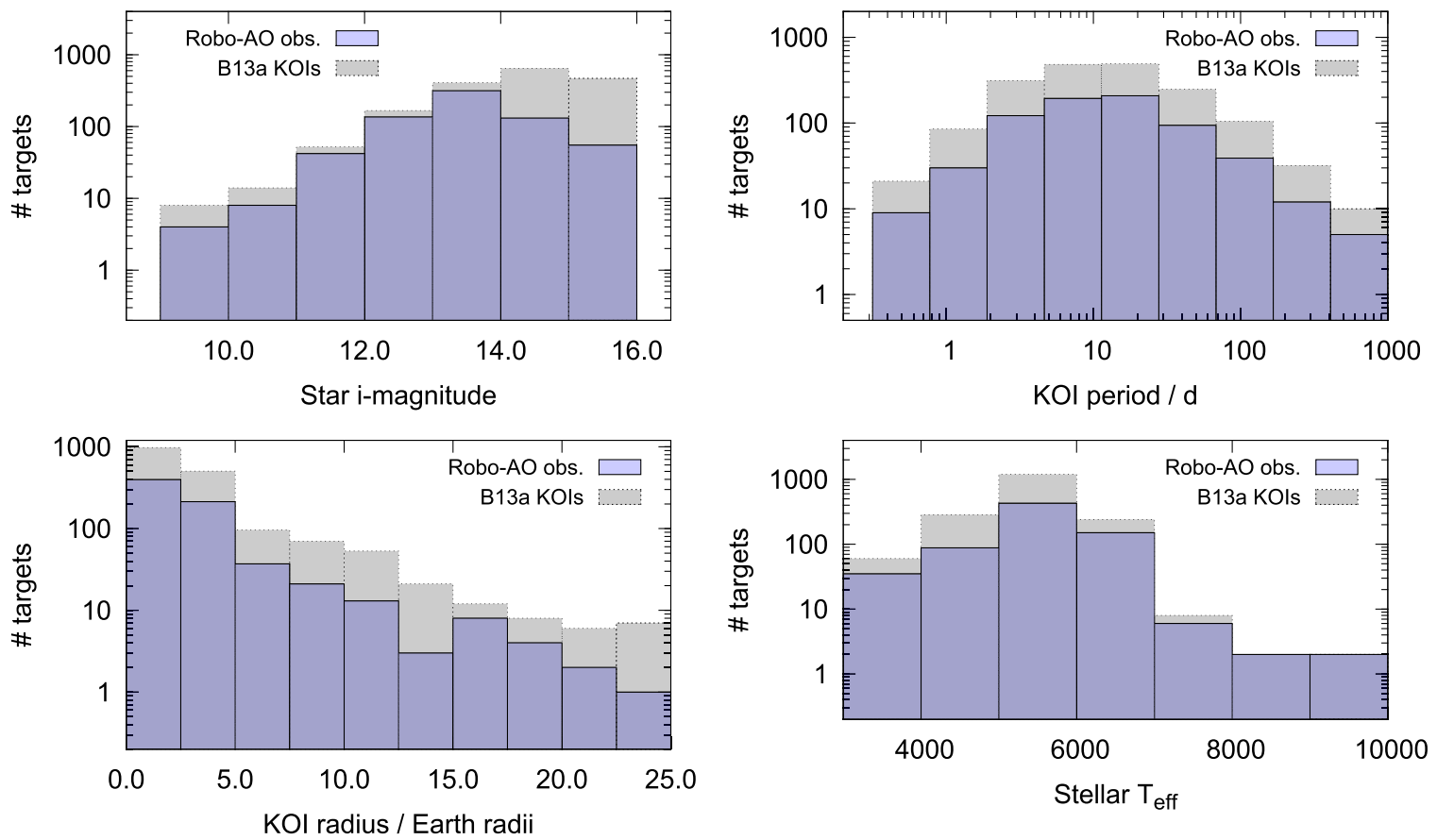

Figure 1. Distribution of the Robo-AO sample compared to the B13a (Batalha et al. 2013) KOIs.

(A color version of this figure is available in the online journal.)

in planetary statistics between different stellar populations, and to generate fully robust statistical studies of the planetary population seen by Kepler.

In order to fully validate the individual Kepler planets and search for correlations between planetary systems and stellar multiplicity properties, we need to search for companions around every KOI. There have been several high-angularresolution surveys of selected samples of KOIs to detect stellar companions and assess the false-positive probability (Adams et al. 2012; Lillo-Box et al. 2012; Horch et al. 2012; Adams et al. 2013; Marcy et al. 2014). However, many of these surveys are performed with adaptive optics systems, and the overheads typically associated with ground-based adaptive optics imaging have limited the number of targets which can be observed.

In this paper, we present the first results from a laser adaptive optics survey that is taking short snapshot high-angularresolution images of every Kepler planet candidate. The survey uses Robo-AO, the first robotic laser adaptive optics system (Baranec et al. 2012, 2013). We designed the automated system for relatively high time-efficiency, allowing the Kepler target list to be completed in $\sim 36 \mathrm{hr}$ of observing time.

This paper presents the 2012-observing-season results of the ongoing Robo-AO KOI survey, covering 715 targets and finding 53 companions, ${ }^{7} 43$ of them new discoveries.

The paper is organized as follows. In Section 2 we describe the Robo-AO system and the KOI survey target selection and observations. Section 3 describes the Robo-AO data reduction and companion-detection pipeline. In Section 4 we describe the survey's results, including the discovered companions. We discuss the results in Section 5, including detailing the effects of the survey's discoveries on the interpretation and veracity of

\footnotetext{
7 For brevity we denote stars which we found within our detection radius of KOIs as "companions," in the sense that they are asterisms associated on the sky. In Section 5 we evaluate the probability that the detected objects are actually physically associated.
}

the observed KOIs, and a brief discussion of the Kepler planet candidates' overall binarity statistics. We conclude in Section 6.

\section{SURVEY TARGETS AND OBSERVATIONS}

\subsection{Target Selection}

We selected targets from the KOIs catalog based on a Q1-Q6 Kepler data search (Batalha et al. 2013). Our initial targets were selected randomly from the Q1-Q6 KOIs, requiring only that the targets are brighter than $m_{i}=16.0$, a restriction which removed only $2 \%$ of the KOIs. While it is our intent to observe every KOI with Robo-AO, this initial target selection provides a wide coverage of the range of KOI properties. Given Robo-AO's low time overheads, we took the time to re-observe KOIs which already had detected companions, to produce a complete and homogenous survey.

In Figure 1 we compare the Robo-AO imaged KOIs to the distribution of all Batalha et al. (2013) KOIs in magnitude, planetary period, planetary radius, and stellar temperature. The Robo-AO list closely follows the KOI list in the range of magnitude covered, with the exception of the three brightest stars (which have already been covered in detail by other non-laser adaptive optics systems), and a reduced coverage of the faintest KOIs, which Robo-AO requires excellent weather conditions to reach. Robo-AO's target distribution closely matches the full KOI list in planetary radius, planetary orbital period, and stellar temperature.

\subsection{Observations}

We obtained high-angular-resolution images of the 715 Kepler targeted planet candidate host stars in summer 2012. We performed all the observations in a queue-scheduled mode with the Robo-AO laser adaptive optics system (Baranec et al. 2012, 2013; Riddle et al. 2012) mounted on the robotic Palomar 60 inch telescope (Cenko et al. 2006). The survey and system specifications are summarized in Table 1. 


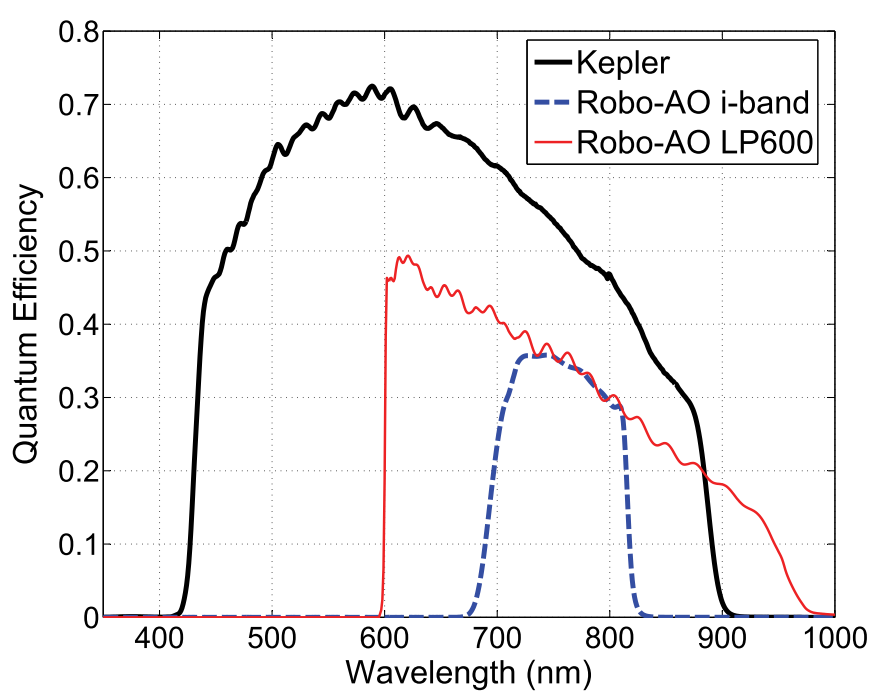

Figure 2. Kepler and Robo-AO passbands. The Robo-AO curves are generated from measured reflection and transmission data from all optical components with the exception of the primary and secondary of the 60 inch telescope which are assumed to be ideal bare aluminium. The Kepler curve is adapted from the Kepler Instrument Handbook.

(A color version of this figure is available in the online journal.)

Table 1

The Specifications of the Robo-AO KOI Survey

\begin{tabular}{lc}
\hline \hline \multicolumn{2}{c}{ KOI Survey Specifications } \\
\hline KOI targets observed & 715 \\
Exposure time & $90 \mathrm{~s}$ \\
Observation wavelengths & $600-950 \mathrm{~nm}$ \\
FWHM resolution & $0 !^{\prime} 12-0^{\prime \prime} 15$ \\
Field of view & $44^{\prime \prime} \times 44^{\prime \prime}$ \\
Pixel scale & 43.1 mas pixel $^{-1}$ \\
Detector format & $1024^{2}$ pixels \\
Detectable magnitude ratio & $\Delta m=5$ mag. at $0^{\prime \prime} 5$ (typical) \\
Observation date range & 2012 Jun $17-2012$ Oct 6 \\
Targets observed/hr & 20 \\
\hline
\end{tabular}

Robo-AO observed the targets between 2012 June 17 and 2012 October 6, on 23 separate nights (detailed in Table 5 in the Appendix). We chose a standardized $90 \mathrm{~s}$ exposure time to provide a snapshot image which would contain all sources likely to affect the Kepler light curve, including close-in sources up to $\sim 5$ mag fainter than the Kepler target. For the observations described here, we used either a Sloan $i^{\prime}$-band filter (York et al. 2000) or a long-pass filter cutting on at $600 \mathrm{~nm}$ (LP600 hereafter). The latter filter roughly matches the Kepler passband (Figure 2) at the redder wavelengths while suppressing the blue wavelengths which have reduced adaptive optics performance (except in the very best seeing conditions). Compared to nearinfrared adaptive optics observations, this filter more closely approximates direct measurement of the effects of unresolved companions on the Kepler light curves.

Two dominant factors affect Robo-AO's imaging performance: the seeing and the brightness of the target. During the 23 nights of observing, the median seeing was 1 ".2, with minimum and maximum values of 0.8 and $1^{\prime \prime} .9$, respectively. We developed an automated routine to measure the actual imaging performance and to classify the targets into the imagingperformance classes given in the full observations list; this classification can be used with the contrast curve for each class to estimate the companion-detection performance for each target (Section 3.4).

\section{DATA REDUCTION}

To search the large data set for companions we developed a fully automated pipeline for data reduction, point-spread function (PSF) subtraction, companion detection and companion measurements in Robo-AO data. The pipeline first takes the short-exposure data cubes recorded by the EMCCD camera and produces dark, flat-field and tip-tilt-corrected co-added output images (Section 3.1). We then subtract a locally optimized PSF estimate from the image of the Kepler target in each field (Section 3.2), and either detect companions around the target stars or place limits on their existence (Section 3.3). Finally, we measure the properties of the detected companions (Section 3.5).

\subsection{Imaging Pipeline}

The Robo-AO imaging pipeline (Law et al. 2012; Terziev et al. 2013) is based on the Lucky Imaging reduction system described in Law et al. (2006a, 2006b, 2009). The recorded EMCCD-frames are dark-subtracted and flat-fielded, and are then corrected for image motion using a bright star in the field. For the KOI observations the relatively crowded fields often led to the automatic selection of a different guide star from the KOI. To avoid having to account for the effects of tip/tilt anisoplanatasism, we manually checked the location of the KOI in Digital Sky Survey images and selected the KOI itself as the guide star in each observation. To produce more consistent and predictable imaging performance for groups of similar KOIs, we used the KOI even if a brighter guide star was nearby and offered potentially increased performance.

\subsection{PSF Subtraction using the Large Set of Robo-AO Target Observations}

The KOI target stars are all in similar parts of the sky, have similar brightness, and were observed at similar airmasses. Because it is unlikely that a companion would be found in the same position for two different targets, we can use each night's ensemble of (at least 20) KOI observations as PSF references without requiring separate observations.

We use a custom locally optimized PSF subtraction routine based on the Locally Optimized Combination of Images algorithm (Lafrenière et al. 2007). For each KOI target we select 20 other KOI observations obtained in the same filter and closest to the target observation in time. We divide the region around the target star into sections based on polar coordinates: five upsampled pixels (110 mas) in radius and $45^{\circ}$ in angle. Similar sections are extracted from each PSF reference image.

We then generate a locally optimized estimate of the PSF in each section by generating linear combinations of the reference PSFs. In each section, an initial PSF is generated by averaging all the reference PSFs. We then use a downhill simplex algorithm to optimize the contribution from each PSF image, searching for the combination which provides the best fit to the target image. This optimization is done on several sections simultaneously (in a region three sections in radius and two sections in angle) to minimize the probability of the algorithm artificially subtracting out real companions. After optimization in the large region, only the central section is output to the final PSF. This provides smooth transitions between adjacent PSF sections because they share many of the image pixels used for the optimization. 

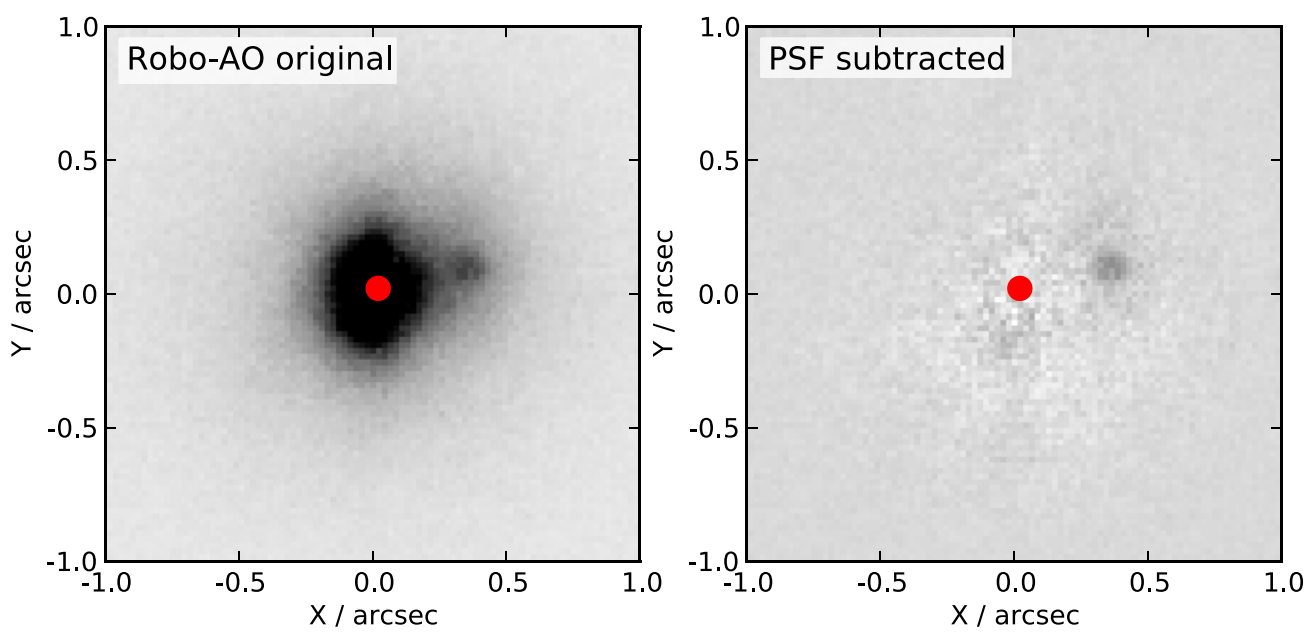

Figure 3. Typical Robo-AO target before and after PSF subtraction using the locally optimized ensemble of PSF references described in the text. The red circle shows the position of the primary star's PSF peak.

(A color version of this figure is available in the online journal.)

This procedure is iterated across all the sections of the image, producing a PSF which is an optimal local combination of the reference PSFs and which can then be subtracted from the target star's PSF. The PSF subtraction typically leaves residuals that are consistent with photon noise only (for these relatively short exposures). Figure 3 shows an example of the PSF subtraction performance.

\subsection{Automated Companion Detection}

We limited the detection radius of this initial search to a 2 .'5 radius from the target KOIs, covering the range of separations between seeing-limited surveys and $\approx 0$.' 15 (subsequent papers will present an analysis of wider-radius companions in Robo-AO imaging).

To more easily and robustly find companions in this large data set, we developed a new automated companion detection algorithm for Robo-AO data. We first measure the local image noise as a function of distance from the target star, by covering the PSF-subtracted target image with four-pixel-diameter apertures and measuring the rms of the pixel values in each aperture, along with the average PSF-subtraction residual signal. We then fit a quadratic to interpolate the changes in noise and residual values as a function of radius from the target star position. For each pixel in the PSF-subtracted image we then use the noise and residual fits to estimate the significance of that pixel's signal level. This procedure generates a significance image where bright pixels in regions of high photon noise (i.e., in the core of the star) are down-weighted compared to those in lower-noise areas.

The significance image yields the pixels which have some chance of denoting detections of stars, but does not take into account the shapes of the detections - a single bright pixel surrounded by insignificant pixels is more likely to be due to a cosmic ray hit than a stellar companion, and a tens-ofpixels-wide blob is likely due to imperfect PSF subtraction. We quantify this by cross-correlating the significance image by a Gaussian corresponding to the diffraction limit of the Robo-AO observation. We then select the pixels which show the most significant detections $(>5 \sigma)$ as possible detections, and amalgamate groups of multiple significant pixels into single detections.
After automated companion detection we also manually checked each image for companions, to check the performance of the automated system and to search for faint but real companions which could have been fit and removed by spurious speckles in the PSF references. The automated system picked up every manually flagged companion, and had a $3.5 \%$ falsepositive rate from all the images, mainly due imperfect PSF subtraction.

\subsection{Imaging Performance Metrics}

We evaluated the contrast-versus-radius detection performance of the PSF-subtraction and automated companion detection code by performing Monte Carlo companion-detection simulations. The time-consuming simulations could only be performed on a group of representative targets, and so we established a quantitative image quality metric that allows each of our observations to be tied into the contrast curves for a particular test target. We first parameterized the performance of each observation of our data set by fitting a two-component model to the PSF based on two Moffat functions tuned to separately measure the widths of the core and halo of the PSF. We then picked 12 single-star observations to represent the variety of PSF parameter space in our data set. For each test star, we added a simulated companion into the observation at a random separation, position angle and contrast, ran the PSF subtraction and automated companion detection routines, and measured the detection significance (if any) of the simulated companion. We repeated this for 1000 simulated companions. ${ }^{8}$ We then binned the simulated detections as a function of separation from the target star, and in each radial bin fit a linear significance-versus-contrast relation. We use the intersection of the fitted relation with a $5 \sigma$ detection to provide the minimum-detectable contrast in each radial bin.

We found that the PSF core size was an excellent predictor of contrast performance, while the halo size did not affect the contrast significantly. The halo is effectively removed by the PSF subtraction, and the contrast is thus chiefly limited by the companion signal-to-noise ratio $(\mathrm{S} / \mathrm{N})$, which scales with the achieved PSF core size (rather than the image FWHM,

\footnotetext{
8 For each simulated companion PSF we removed the central spike introduced by shifted-and-added photon-noise-limited detectors by averaging with nearby pixels (Law et al. 2006b, 2009); this conservative correction reduces our claimed detectable contrast by up to $25 \%$.
} 


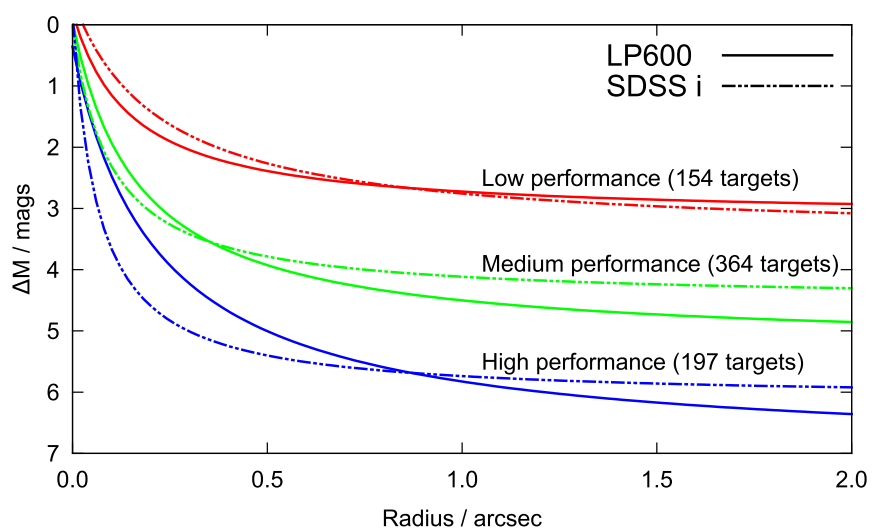

Figure 4. Detectable magnitude ratios for three representative targets observed in the LP600 and SDSS $i^{\prime}$ filters (smoothed with fitting curves generated as described in Section 3.4).

(A color version of this figure is available in the online journal.)

which we found is a weak predictor of contrast performance in Robo-AO data). On this basis, we use the PSF core size to assign targets to contrast-performance groups (low, medium and high). As the imaging-performance degrades, we found that the relative contribution of the fitted core PSF decreases, while the core itself shrinks. The somewhat counter-intuitive size decrease is because poor imaging quality inevitably corresponds to poor $\mathrm{S} / \mathrm{N}$ on the shift-and-add image alignment used by Robo-AO's EMCCD detector. This leads to the frame alignments locking onto photon noise spikes, and thus produces a single-pixel-sized spike in the images (Law et al. 2006b, 2009). We therefore assign images with a diffraction-limited-sizes core ( $\sim 0^{\prime}$. 15) to the high-performance groups; smaller cores, where the imaging performance is degraded, were assigned to the lower-performance groups.

Figure 4 shows the contrast curves resulting from this procedure, for clarity smoothed with fitting functions of form $a-b /(r-c)$ (where $r$ is the radius from the target star and $a$, $b$, and $c$ are fitting variables). The $i$-band observations obtain better contrast close-in than the LP600 filter, because of their improved Strehl ratios, while the broader LP600 filter allows somewhat improved contrast at wider radii under all but the poorest conditions.

\subsection{Companion Characterization}

\subsubsection{Contrast Ratios}

We determined the binaries' contrast ratio in two ways: for the widest separations we performed aperture photometry on the original images; for the closer systems we used the estimated PSF to remove the blended contributions of each of the stars before performing aperture photometry. In all cases the aperture sizes were optimized for the system separation and the available signal.

The locally optimized PSF subtraction will attempt to remove flux associated with companions by using other PSFs with (non-astrophysical) excess brightness in those areas, because it is trying to achieve the best fit to the target images without discrimination between real companions and speckles. By selecting an optimization over a region containing many PSF core sizes, we reduce the algorithm's ability to subtract away companion light for detection purposes. However, the companion will still be artificially faint in PSF-subtracted images, leading to errors in flux ratio measurements. To avoid this, we re-run the PSF fit excluding a six-pixel-diameter region around any detected companion. The PSF-fit regions are large enough to provide a good estimate for the PSF underneath the companion, and the companion brightness is not artificially reduced by this procedure.

We calculated the contrast ratio uncertainty on the basis of the difference between the injected and measured contrasts of the fake companions injected during the contrast-curve calculations (Section 3.3). We found that the detection significance of the companion was the best predictor of the contrast ratio accuracy, and so we use a fit to that relation to estimate the contrast ratio uncertainty for each companion. We note that the uncertainties $(5 \%-30 \%)$ are much higher than would be naively expected from the $\mathrm{S} / \mathrm{N}$ of the companion detection, as they include an estimate of the systematic errors resulting from the AO imaging, $\mathrm{PSF}$-subtraction and contrast-measurement processes.

\subsubsection{Separations and Position Angles}

To obtain the separation and position angle of the binaries we centroided the PSF-subtracted images of the companion and primary, as above. We converted the raw pixel positions to on-sky separations and position angles using a distortion solution produced from Robo-AO measurements of globular clusters observed during the same timeframe as the Robo-AO KOI survey. ${ }^{9}$

We calculated the uncertainties of the companion separation and position angles using estimated systematic errors in the position measurements due to blending between components, depending on the separation of the companion (typically 1-2 pixels uncertainty in the position of each star). We also included an estimate of the maximal changes in the Robo-AO orientation throughout the observation period $( \pm 1.5)$, as verified using the globular cluster measurements above. Finally, we verified the measured positions and contrast ratios in direct measurement from non-PSF-subtracted images.

\section{DISCOVERIES}

We resolved 53 Kepler planet candidate hosts into multiple stars; the discovery images are summarized in Figure 5 and the separations and contrast ratios are shown in Figure 6. Section 5 addresses the probability of physical association for these objects. The measured companion properties for the targets with secure detections are detailed in Table 2. Table 3 describes 15 probable companions which fell just below our formal $5 \sigma$ detection criteria. We consider these very likely to be real (indeed, three have been previously detected by other groups), but in the present data we cannot exclude the possibility that one or two of these detections are spurious speckles.

Two of the targets showed potential companions that were not well-resolved by Robo-AO but were suggestive of interesting companions. KOI-1962 showed PSF-core-elongation indicative of a $<0$.' 15 separation nearly equal-magnitude binary. KOI-1964 has a probable faint companion at a separation of 0.4 ; dynamic speckle noise reduces the detection significance to $\approx 3 \sigma$. We confirmed the Robo-AO detections with NIRC2-NGS (Wizinowich et al. 2000) on Keck II on 2013 July 23 (Figure 7).

\subsection{Comparison to Other Surveys}

Lillo-Box et al. (2012, hereafter L12) observed 98 KOIs using a Lucky Imaging system. Seven of the targets for which

\footnotetext{
9 S. Hildebrandt (2013, private communication).
} 
Table 2

Secure Detections of Objects within 2".5 of Kepler Planet Candidates

\begin{tabular}{|c|c|c|c|c|c|c|c|c|}
\hline KOI & $m_{i}$ & $\begin{array}{l}\text { ObsID } \\
\text { (mag) }\end{array}$ & Filter & $\begin{array}{c}\text { Signf. } \\
\sigma\end{array}$ & $\begin{array}{l}\text { Separation } \\
\text { (arcsec) }\end{array}$ & $\begin{array}{l}\text { P.A. } \\
\text { (deg.) }\end{array}$ & $\begin{array}{l}\text { Mag. Diff. } \\
\text { (mag) }\end{array}$ & Previous Detection? \\
\hline KOI-1 & 11.2 & 2012 Jul 16 & $i$ & 13 & $1.13 \pm 0.06$ & $135 \pm 2$ & $3.95 \pm 0.33$ & D09 \\
\hline KOI-13 & 10.5 & 2012 Oct 6 & $i$ & 950 & $1.16 \pm 0.06$ & $279 \pm 2$ & $0.19 \pm 0.06$ & $\mathrm{H} 11, \mathrm{~A} 12$ \\
\hline KOI-98 & 12.0 & 2012 Jul 17 & $i$ & 80 & $0.29 \pm 0.06$ & $140 \pm 6$ & $0.76 \pm 0.16$ & B11, H11, A12, H12 \\
\hline KOI-119 & 12.5 & 2012 Jul 16 & $i$ & 38 & $1.05 \pm 0.06$ & $118 \pm 2$ & $0.87 \pm 0.22$ & \\
\hline KOI-141 & 13.4 & 2012 Jul 18 & $i$ & 34 & $1.10 \pm 0.06$ & $11 \pm 2$ & $1.39 \pm 0.23$ & A12 \\
\hline KOI-162 & 13.6 & 2012 Jul 18 & LP600 & 19 & $0.29 \pm 0.06$ & $117 \pm 7$ & $0.81 \pm 0.29$ & \\
\hline KOI-174 & 13.4 & 2012 Jul 18 & LP600 & 7 & $0.60 \pm 0.06$ & $77 \pm 3$ & $4.43 \pm 0.44$ & A13 \\
\hline KOI-177 & 13.0 & 2012 Jul 18 & $i$ & 12 & $0.24 \pm 0.06$ & $215 \pm 8$ & $0.97 \pm 0.35$ & \\
\hline KOI-191 & 14.7 & 2012 Sep 1 & LP600 & 5 & $1.69 \pm 0.06$ & $94 \pm 2$ & $3.09 \pm 0.49$ & \\
\hline KOI-268 & & 2012 Sep 14 & LP600 & 23 & $1.81 \pm 0.06$ & $265 \pm 2$ & $3.82 \pm 0.27$ & $\mathrm{~A} 12$ \\
\hline KOI-356 & 13.5 & 2012 Jul 28 & LP600 & 17 & $0.56 \pm 0.06$ & $218 \pm 4$ & $2.92 \pm 0.30$ & \\
\hline KOI-401 & 13.7 & 2012 Aug 5 & LP600 & 19 & $1.99 \pm 0.06$ & $268 \pm 2$ & $2.90 \pm 0.29$ & $\mathrm{~L} 12$ \\
\hline KOI-511 & 14.0 & 2012 Sep 1 & LP600 & 7 & $1.28 \pm 0.06$ & $123 \pm 2$ & $3.33 \pm 0.43$ & \\
\hline KOI-640 & 13.1 & 2012 Jul 28 & $i$ & 16 & $0.44 \pm 0.06$ & $117 \pm 4$ & $0.62 \pm 0.31$ & \\
\hline KOI-687 & 13.6 & 2012 Aug 4 & $i$ & 21 & $0.70 \pm 0.06$ & $13 \pm 3$ & $2.04 \pm 0.28$ & \\
\hline KOI-688 & 13.8 & 2012 Sep 14 & LP600 & 19 & $1.71 \pm 0.06$ & $141 \pm 2$ & $2.19 \pm 0.29$ & \\
\hline KOI-712 & 13.5 & 2012 Aug 5 & $i$ & 21 & $0.47 \pm 0.06$ & $173 \pm 4$ & $1.17 \pm 0.28$ & \\
\hline KOI-984 & 11.4 & 2012 Aug 3 & $i$ & 120 & $1.80 \pm 0.06$ & $42 \pm 2$ & $0.01 \pm 0.14$ & \\
\hline KOI-1002 & 13.4 & 2012 Aug 3 & $i$ & 9 & $0.30 \pm 0.06$ & $173 \pm 6$ & $2.31 \pm 0.38$ & \\
\hline KOI-1050 & 13.7 & 2012 Aug 3 & $i$ & 8 & $2.09 \pm 0.06$ & $197 \pm 2$ & $2.70 \pm 0.40$ & \\
\hline KOI-1150 & 13.1 & 2012 Aug 5 & $i$ & 9 & $0.39 \pm 0.06$ & $322 \pm 5$ & $2.41 \pm 0.39$ & \\
\hline KOI-1152 & 13.6 & 2012 Sep 14 & LP600 & 16 & $0.59 \pm 0.06$ & $2 \pm 3$ & $0.31 \pm 0.31$ & \\
\hline KOI-1274 & 13.1 & 2012 Aug 6 & $i$ & 7 & $1.10 \pm 0.06$ & $241 \pm 2$ & $3.75 \pm 0.44$ & \\
\hline KOI-1613 & & 2012 Aug 29 & $i$ & 36 & $0.22 \pm 0.06$ & $184 \pm 9$ & $1.30 \pm 0.22$ & \\
\hline KOI-1619 & 11.4 & 2012 Aug 29 & $i$ & 60 & $2.10 \pm 0.06$ & $226 \pm 2$ & $2.82 \pm 0.18$ & \\
\hline KOI-1677 & 14.1 & 2012 Sep 4 & LP600 & 7 & $0.61 \pm 0.06$ & $159 \pm 3$ & $4.76 \pm 0.44$ & \\
\hline KOI-1880 & 13.8 & 2012 Jul 15 & LP600 & 6 & $1.70 \pm 0.06$ & $100 \pm 2$ & $3.66 \pm 0.45$ & \\
\hline KOI-1890 & 11.6 & 2012 Aug 29 & $i$ & 42 & $0.41 \pm 0.06$ & $142 \pm 5$ & $3.44 \pm 0.21$ & \\
\hline KOI-1916 & 13.4 & 2012 Sep 13 & LP600 & 31 & $0.27 \pm 0.06$ & $143 \pm 7$ & $2.73 \pm 0.24$ & \\
\hline KOI-1962 & & 2012 Aug 30 & $i$ & $\ldots$ & $0.12 \pm 0.03$ & $\ldots$ & $0.04\left(K_{s}\right)$ & \\
\hline KOI-1964 & 10.5 & 2012 Aug 30 & $i$ & $\ldots$ & $0.39 \pm 0.03$ & $\ldots$ & $1.9\left(K_{S}\right)$ & \\
\hline KOI-1979 & 12.8 & 2012 Aug 30 & $i$ & 9 & $0.84 \pm 0.06$ & $192 \pm 3$ & $3.20 \pm 0.39$ & \\
\hline KOI-2059 & 12.6 & 2012 Oct 6 & LP600 & 120 & $0.38 \pm 0.06$ & $291 \pm 5$ & $1.10 \pm 0.14$ & \\
\hline KOI-2143 & 13.9 & 2012 Oct 6 & LP600 & 19 & $2.16 \pm 0.06$ & $317 \pm 2$ & $3.50 \pm 0.29$ & \\
\hline KOI-2463 & 12.6 & 2012 Aug 31 & $i$ & 70 & $0.62 \pm 0.06$ & $125 \pm 3$ & $0.75 \pm 0.17$ & \\
\hline KOI-2486 & 12.9 & 2012 Aug 31 & $i$ & 18 & $0.24 \pm 0.06$ & $63 \pm 8$ & $0.49 \pm 0.30$ & \\
\hline KOI-2641 & 13.6 & 2012 Oct 6 & LP600 & 36 & $1.42 \pm 0.06$ & $214 \pm 2$ & $2.56 \pm 0.22$ & \\
\hline KOI-2657 & 12.7 & 2012 Oct 6 & LP600 & 62 & $0.73 \pm 0.06$ & $131 \pm 3$ & $0.27 \pm 0.18$ & \\
\hline
\end{tabular}

Notes. References for previous detections are denoted with the following codes: Adams et al. 2012 (A12); Adams et al. 2013 (A13); Buchhave et al. 2011 (B11); Daemgen et al. 2009 (D09); Horch et al. 2012 (H12); Howell et al. 2011 (H11); Lillo-Box et al. 2012 (L12).

Table 3

Likely Detections of Objects within 2".5 of Kepler Planet Candidates

\begin{tabular}{|c|c|c|c|c|c|c|c|c|}
\hline KOI & $m_{i}$ & $\begin{array}{l}\text { ObsID } \\
\text { (mag) }\end{array}$ & Filter & $\begin{array}{l}\text { Signf. } \\
\sigma\end{array}$ & $\begin{array}{l}\text { Separation } \\
(\operatorname{arcsec})\end{array}$ & $\begin{array}{l}\text { P.A. } \\
\text { (deg.) }\end{array}$ & $\begin{array}{l}\text { Mag. Diff. } \\
\text { (mag) }\end{array}$ & Previous Detection? \\
\hline KOI-97 & 12.7 & 2012 Jul 17 & $i$ & 4.2 & $1.90 \pm 0.06$ & $99 \pm 2$ & $4.61 \pm 0.52$ & A12 \\
\hline KOI-306 & 12.4 & 2012 Jul 18 & $i$ & 3.6 & $2.06 \pm 0.06$ & $243 \pm 2$ & $4.16 \pm 0.56$ & A12 \\
\hline KOI-628 & 13.7 & 2012 Aug 3 & $i$ & 1.4 & $1.83 \pm 0.06$ & $309 \pm 2$ & $5.20 \pm 0.80$ & L12 \\
\hline KOI-987 & 12.3 & 2012 Aug 3 & $i$ & 2.4 & $2.05 \pm 0.06$ & $225 \pm 2$ & $4.10 \pm 0.66$ & \\
\hline KOI-1151 & 13.2 & 2012 Aug 5 & $i$ & 3.2 & $0.75 \pm 0.06$ & $309 \pm 3$ & $3.49 \pm 0.58$ & \\
\hline KOI-1359 & 15.0 & 2012 Sep 4 & LP600 & 3.4 & $1.43 \pm 0.06$ & $333 \pm 2$ & $3.80 \pm 0.57$ & \\
\hline KOI-1375 & 13.5 & 2012 Aug 6 & $i$ & 4.0 & $0.77 \pm 0.06$ & $269 \pm 3$ & $4.38 \pm 0.53$ & \\
\hline KOI-1442 & 12.3 & 2012 Aug 6 & $i$ & 3.3 & $2.24 \pm 0.06$ & $70 \pm 2$ & $6.68 \pm 0.57$ & \\
\hline KOI-1845 & 14.1 & 2012 Sep 13 & LP600 & 2.9 & $2.06 \pm 0.06$ & $77 \pm 2$ & $4.97 \pm 0.60$ & \\
\hline KOI-1884 & 15.2 & 2012 Sep 13 & LP600 & 2.5 & $0.95 \pm 0.06$ & $96 \pm 2$ & $3.65 \pm 0.64$ & \\
\hline KOI-1891 & 15.0 & 2012 Sep 13 & LP600 & 3.0 & $2.09 \pm 0.06$ & $210 \pm 2$ & $4.46 \pm 0.60$ & \\
\hline KOI-2009 & 13.6 & 2012 Sep 14 & LP600 & 4.9 & $1.51 \pm 0.06$ & $176 \pm 2$ & $4.11 \pm 0.49$ & \\
\hline KOI-2159 & 13.3 & 2012 Aug 31 & $i$ & 4.0 & $2.00 \pm 0.06$ & $323 \pm 2$ & $3.99 \pm 0.53$ & \\
\hline KOI-2413 & 14.7 & 2012 Sep 14 & LP600 & 2.4 & $0.31 \pm 0.06$ & $67 \pm 6$ & $2.11 \pm 0.66$ & \\
\hline KOI-2443 & 13.8 & 2012 Oct 6 & LP600 & 3.7 & $1.39 \pm 0.06$ & $163 \pm 2$ & $5.37 \pm 0.55$ & \\
\hline
\end{tabular}

Notes. References for previous detections are denoted with the following codes: Adams et al. 2012 (A12); Lillo-Box et al. 2012 (L12). 


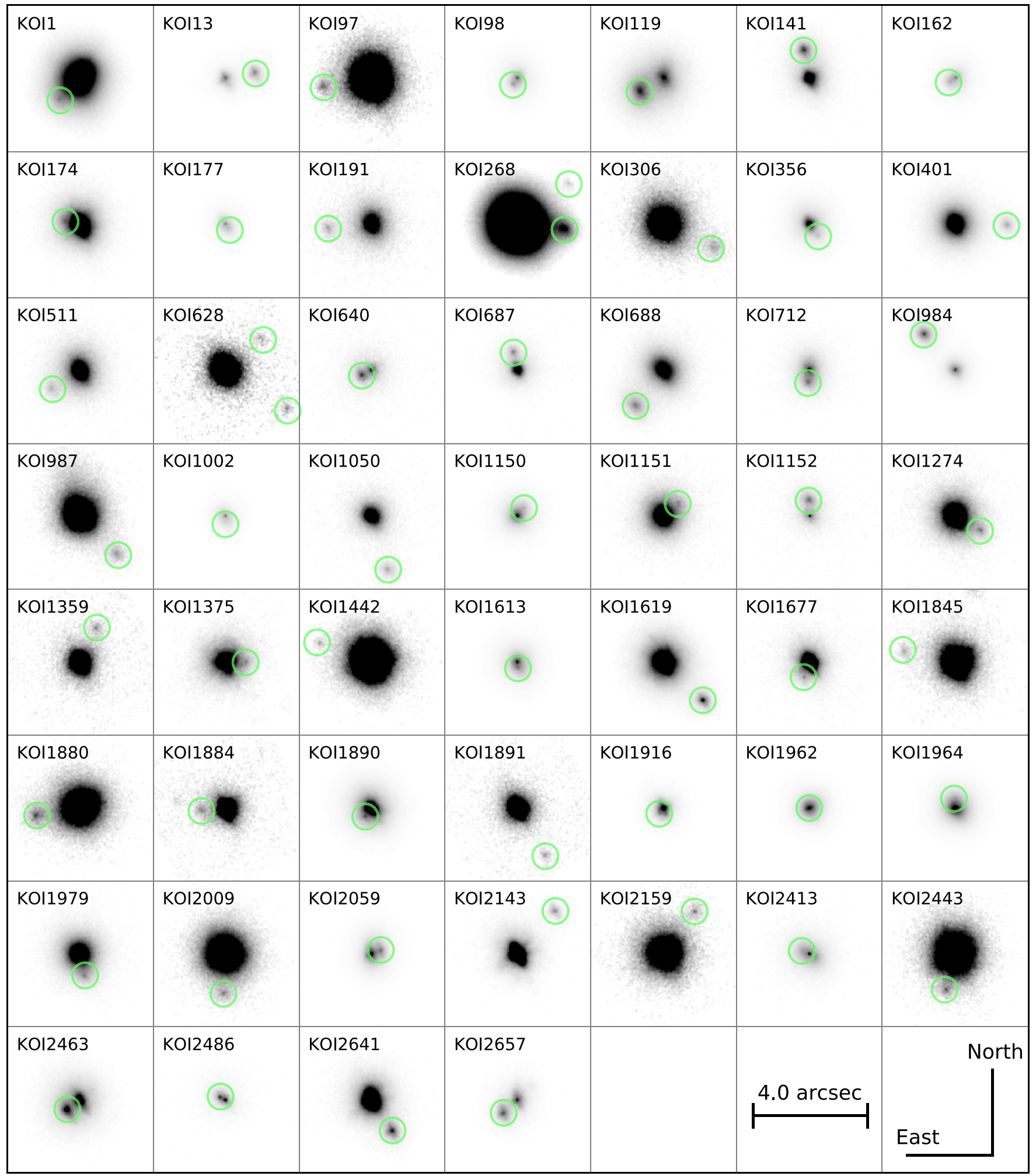

Figure 5. Kepler planet candidates resolved into multiple stars by Robo-AO. The grayscale of each $4^{\prime \prime}$ cutout is selected to show the companion; the angular scale and orientation is identical for each cutout.

(A color version of this figure is available in the online journal.)

they discovered companions within a 2".5 radius are also in our survey. Both surveys detect KOI-401 at a separation of 2."0 and at a contrast of $2.6 \mathrm{mag}$ (L12 $i$-band) or 2.9 mag (Robo-AO LP600). The companions to KOI-628 were visible in our survey but at contrasts that placed them in the "likely detections" group. L12 detected a companion to KOI-658 at 1".9 radius and a contrast of 4.6 mag in $i$-band. At that radius, for the performance achieved on KOI-658, the Robo-AO snapshot-survey limiting magnitude ratio is $\sim 4.0 \mathrm{mag}$ and so we do not re-detect that companion. For the same reason we also do not re-detect the companions to KOI-703 (6.4 mag contrast), KOI-704 (5.0 mag contrast) and KOI-721 (3.9 mag contrast). The 0'.13 radius 


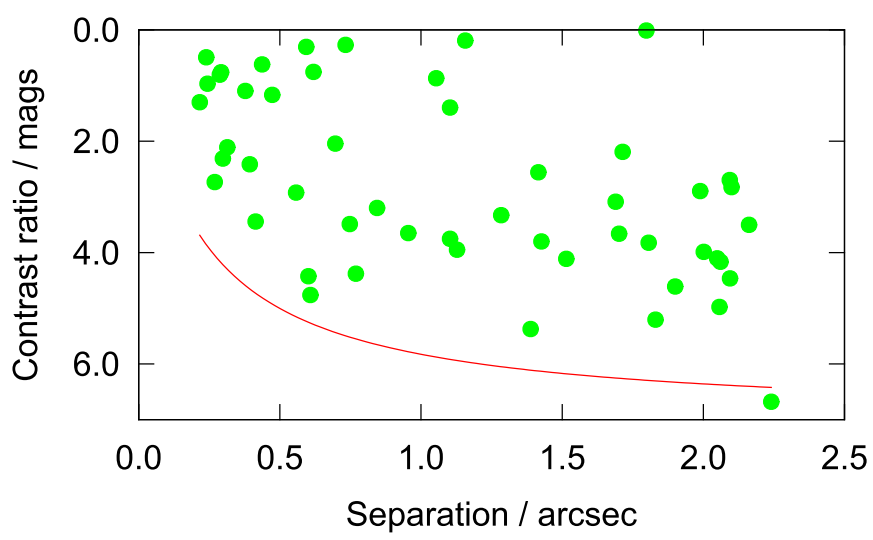

Figure 6. Separations and magnitude differences of the detected companions compared to the survey's typical high-performance $5 \sigma$ contrast curve (one very faint companion was detected around a bright KOI in exceptional conditions). The distribution of companion properties has no evidence for unaccounted incompleteness effects, although there is an excess of bright companions at close separations, suggesting that those companions are more likely to be physically associated.

(A color version of this figure is available in the online journal.)

companion to KOI-1537 detected in Adams et al. (2013) is at too close a separation to be detectable in our survey. The L12 companion to KOI-1375 is visible in our data set, but has a contrast ratio of $4.0 \mathrm{mag}$, under our formal detection limit and well below the 2.75 mag $i$-band contrast measured by L12. The target is not strongly colored according to L12 and it is not obvious why the companion is so much fainter in our survey.

\section{DISCUSSION}

\subsection{Implications for Kepler Planet Candidates}

The detection of a previously unknown star within the photometric aperture of a KOI host star will affect the derived radius of any planet candidate around that host star, because the Kepler observed transit depth is shallower than the true depth due to dilution. The degree of this effect depends upon the relative brightness of the target and secondary star, and which star is actually being transited. In particular, if there is more than one star in the photometric aperture and the transiting object is around a star that contributes a fraction $F_{i}$ to the total light in the aperture, then

$$
\delta_{\text {true }}=\delta_{\text {obs }}\left(\frac{1}{F_{i}}\right)
$$

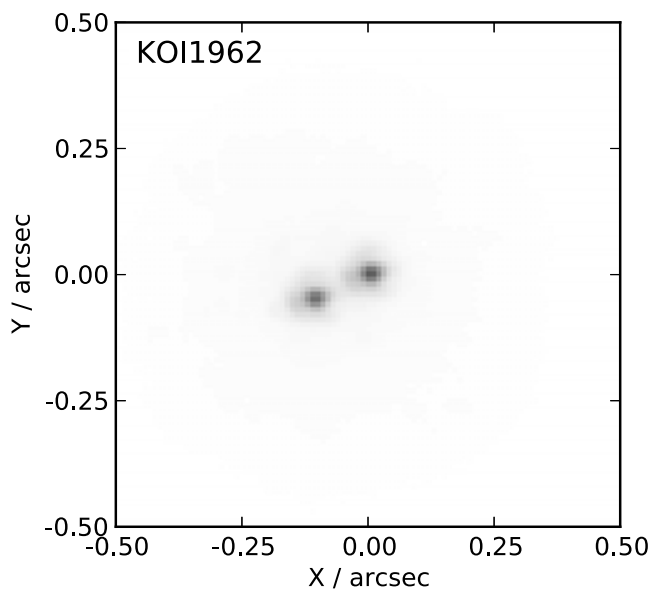

Figure 7. Keck-AO NIRC2 J-band images confirming two Robo-AO companion detections.

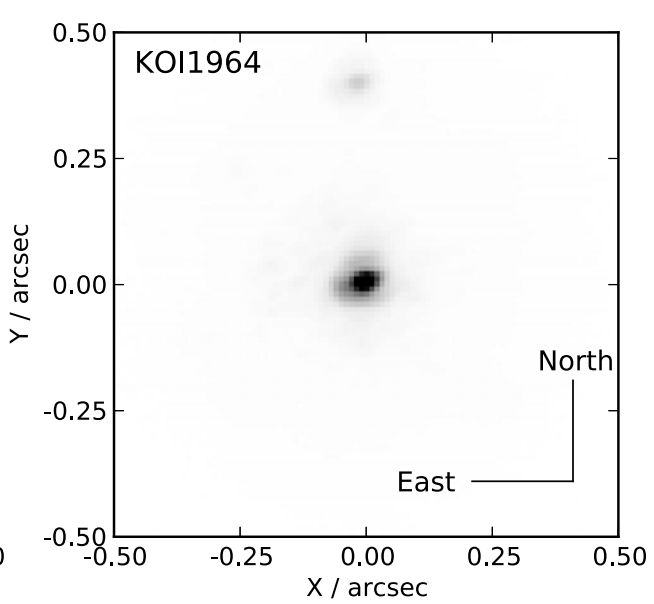

where $\delta_{\text {true }}$ is the true intrinsic fractional transit depth and $\delta_{\text {obs }}$ is the observed, diluted depth. Since $\delta \propto\left(R_{p} / R_{\star}\right)^{2}$, the true planet radius in the case where the transit is around star $i$ is

$$
R_{p, i}=R_{\star, i}\left(\frac{R_{p}}{R_{\star}}\right)_{0} \sqrt{\frac{1}{F_{i}}},
$$

where $R_{\star, i}$ is the radius of star $i$, and the 0 subscript represents the radius ratio implied by the diluted transit, or what would be inferred by ignoring the presence of any blending flux.

Thus, for each planet candidate in KOI systems observed to have close stellar companions, the derived planet radius must be corrected-and there are two potential scenarios for each candidate: the eclipsed star is either star A (the brighter target star) or star B (the fainter companion).

In case $A$, the corrected planet radius is

$$
R_{p, A}=R_{p, 0} \sqrt{\frac{1}{F_{A}}},
$$

and in case B,

$$
R_{p, B}=R_{p, 0} \frac{R_{B}}{R_{A}} \sqrt{\frac{1}{F_{B}}} .
$$

Case A is straightforward, with nothing needed except the observed contrast ratio (in order to calculate $F_{A}$ ). It should be noted, however, that this assumes that the estimated host stellar radius $R_{A}$ is unchanged by the detection of the companion star. As the radii for most Kepler stars are inferred photometrically, this may not be strictly true, as light from the companion might cause the primary stellar type to be misidentified. We do not attempt to quantify the extent of this effect in this paper. We do, however, note that it is likely to be negligible for larger contrast ratios where the colors of the blended system are dominated by light from the primary.

Case $\mathrm{B}$, in addition to needing $F_{B}$, needs also the ratio $R_{B} / R_{A}$. If the observed companion is an unassociated background star, then the single-band Robo-AO observation does not constrain $R_{B}$. However, under the assumption that the companion is physically bound, then we can estimate its size and spectral type, given assumed knowledge about the primary star A.

In order to accomplish this, we use the Dartmouth stellar models (Dotter et al. 2008) and the measured primary KOI star 
Table 4

Implications on Derived Radius of Kepler Planet Candidates

\begin{tabular}{|c|c|c|c|c|c|c|c|c|c|}
\hline $\begin{array}{l}\text { KOI } \\
-\end{array}$ & $\begin{array}{l}P^{\mathrm{a}} \\
\text { (d) }\end{array}$ & $\begin{array}{c}R_{p}{ }^{\mathrm{a}} \\
\left(R_{\oplus}\right)\end{array}$ & $\begin{array}{c}R_{\star}^{\mathrm{a}} \\
\left(R_{\odot}\right)\end{array}$ & $\begin{array}{c}\Delta m \\
(\mathrm{mag})\end{array}$ & $\begin{array}{r}\text { sep } \\
\left({ }^{\prime \prime}\right)\end{array}$ & $\begin{array}{l}R_{\star, B}{ }^{\mathrm{b}} \\
\left(R_{\odot}\right)\end{array}$ & $\begin{array}{c}R_{p, A}{ }^{\mathrm{c}} \\
\left(R_{\oplus}\right)\end{array}$ & $\begin{array}{l}R_{p, B} \\
\left(R_{\oplus}\right)\end{array}$ & $\begin{array}{r}R_{p, B_{b g}}{ }^{\mathrm{d}} \\
\quad\left(R_{\oplus}\right)\end{array}$ \\
\hline 1.01 & $2.4 / 1$ & 14.40 & 1.06 & 4.0 & 1.15 & 0.50 & 14.0 & 42.0 & 84.9 \\
\hline 13.01 & .764 & 23.00 & 2.70 & 0.2 & 1.16 & 2.70 & 31.2 & 34.0 & 12.6 \\
\hline 7.01 & 4.885 & 16.10 & 1.78 & 4.6 & 1.90 & 0.57 & 16.2 & 43.6 & 76.1 \\
\hline 8.01 & 6.790 & 10.00 & 1.63 & 0.8 & 0.29 & 1.26 & 12.2 & 3.4 & 10.7 \\
\hline 119.01 & 49.184 & 3.90 & 0.94 & 0.9 & 1.05 & 0.76 & 4.7 & 5.6 & 7.5 \\
\hline 119.02 & 190.313 & 3.40 & $\ldots$ & . & . & . . & 4.1 & 4.9 & 6.5 \\
\hline 141.01 & 2.624 & 5.43 & 0.93 & 1.4 & 1.10 & 0.72 & 6.1 & 9.0 & 12.5 \\
\hline 162.01 & 14.006 & 2.54 & 0.96 & 0.8 & 0.29 & 0.79 & 3.1 & 3.7 & 4.7 \\
\hline 174.01 & 56.354 & 1.94 & 0.63 & 4.4 & 0.60 & 0.21 & 2.0 & 5.1 & 24.0 \\
\hline 177.01 & 21.060 & 1.84 & 1.06 & 1.0 & 0.24 & 0.84 & 2.2 & 2.7 & 3.2 \\
\hline 191.01 & 15.359 & 11.00 & 0.88 & 3.1 & 1.69 & 0.55 & 11.3 & 29.3 & 53.4 \\
\hline 191.02 & 2.418 & 2.30 & & . & .. & $\ldots$ & 2.4 & 6.1 & 11.2 \\
\hline 191.03 & 0.709 & 1.24 & & & $\ldots$ & . & 1.3 & 3.3 & 6.0 \\
\hline 191.04 & 38.652 & 2.30 & $\ldots$ & $\ldots$ & $\ldots$ & $\ldots$ & 2.4 & 6.1 & 11.2 \\
\hline 268.01 & 110.379 & 1.73 & 0.79 & 3.8 & 1.81 & 0.33 & 1.8 & 4.3 & 13.0 \\
\hline 306.01 & 24.308 & 2.29 & 0.87 & 4.2 & 2.06 & 0.39 & 2.3 & 7.0 & 18.0 \\
\hline 356.01 & 1.827 & 5.73 & 1.60 & 2.9 & 0.56 & 0.66 & 5.9 & 9.4 & 14.2 \\
\hline 01.01 & 29.199 & 7.23 & 1.58 & 2.9 & 1.99 & 0.66 & 7.5 & 11.9 & 18.0 \\
\hline 401.02 & 160.017 & 7.31 & $\ldots$ & & & $\ldots$ & 7.6 & 2.0 & 18.2 \\
\hline 401.03 & .328 & 2.66 & $\ldots$ & & $\ldots$ & $\ldots$ & & 4.4 & 6.6 \\
\hline 511.01 & 006 & 2.80 & 1.08 & 3.3 & 1.28 & 0.61 & 2.9 & 7.5 & 12.3 \\
\hline 511.02 & 4.264 & 1.58 & $\ldots$ & $\ldots$ & $\ldots$ & $\ldots$ & 1.6 & 4.2 & 6.9 \\
\hline 28.01 & 14.486 & 3.10 & 1.29 & 5.2 & 1.83 & 0.38 & 3.1 & 10.1 & 26.5 \\
\hline 40.01 & 30.996 & 2.44 & 0.89 & 0.6 & 0.44 & 0.80 & 3.1 & 3.6 & 4.5 \\
\hline 8701 & 78 & 1.46 & 0.93 & 2.0 & 0.70 & 0.64 & 1 & 2.8 & 4.3 \\
\hline 688.01 & 3.276 & 2.28 & 1.35 & 2.2 & 1.71 & 0.77 & 2.4 & 3.8 & 4.9 \\
\hline 712.01 & 2.178 & 1.08 & 0.84 & 1.2 & 0.47 & 0.76 & 1.3 & 1.9 & 2.6 \\
\hline 984.01 & 4.287 & 3.19 & 0.92 & 0.0 & 1.80 & 0.92 & 4.5 & 4.5 & 4.9 \\
\hline & & 1.28 & 0.92 & & 2.05 & 0.42 & & & 9.3 \\
\hline 1002.01 & .482 & 1.36 & 1.01 & & 0.30 & 0.66 & 1 & 2.7 & 4.1 \\
\hline 1050.01 & 1.269 & 1.40 & 0.76 & 2.7 & 2.09 & 0.46 & 1. & 3.1 & 6.6 \\
\hline 1050.02 & 2.853 & 1.40 & $\ldots$ & $\ldots$ & $\ldots$ & $\ldots$ & 1.5 & 3.1 & 6.6 \\
\hline 1150.01 & 0.677 & 1.10 & 1.09 & 2.4 & 0.39 & 0.66 & 1.2 & 2.1 & 3.2 \\
\hline & & 1.46 & 0.97 & 3.5 & 0.75 & 0.50 & & & 7.7 \\
\hline 1151.02 & 411 & 1.15 & $\cdots$ & $\cdots$ & . & $\ldots$ & 1. & 3.0 & 6.0 \\
\hline 1151.03 & 5.249 & 0.70 & $\ldots$ & & $\ldots$ & . & 0.7 & 1.8 & 3.7 \\
\hline 1151.04 & 17.453 & 0.87 & . & & $\ldots$ & & 0.9 & 2.3 & 4.6 \\
\hline 1151.05 & 21.720 & 0.97 & & & & & & 2.5 & 5.1 \\
\hline 52.01 & 4.722 & 19.56 & 0.65 & 0.3 & 0.59 & 0.54 & & 4.9 & 45.7 \\
\hline 1274.01 & 362.000 & 4.73 & 0.79 & 3.8 & 1.10 & 0.37 & 48 & 2.6 & 34.3 \\
\hline 1359.01 & 37.101 & 3.50 & 0.92 & 3.8 & 1.43 & 0.58 & 3.6 & 13.0 & 22.2 \\
\hline 1359.02 & 104.820 & 7.30 & $\ldots$ & $\ldots$ & $\ldots$ & & 7.4 & 27.1 & 46.3 \\
\hline 1375.01 & 321.214 & 6.78 & 1.17 & & 0.77 & 0.50 & 6 & .0 & 4.0 \\
\hline 1442.01 & 0.669 & 1.23 & 1.00 & 6.7 & 2.24 & 0.20 & 1 & 5.2 & 26.8 \\
\hline 1613.01 & 15.866 & 1.07 & 1.04 & 1.3 & 0.22 & 0.78 & 1 . & 1.7 & 2.1 \\
\hline 1613.02 & 94.091 & 1.08 & $\ldots$ & $\ldots$ & $\ldots$ & $\ldots$ & 1.2 & 1.7 & 2.2 \\
\hline 1619.01 & 20.666 & 0.80 & 0.62 & 2.8 & 2.10 & 0.33 & 0 & 1.6 & 4.9 \\
\hline 1677.01 & & 2.18 & 0.85 & 4.8 & 0.61 & 0.43 & 2. & 9.8 & 23.1 \\
\hline 1677.02 & 8.512 & 0.81 & $\ldots$ & $\ldots$ & $\ldots$ & & 0.8 & 3.7 & 8.6 \\
\hline 1845.01 & 1.970 & 1.50 & 0.70 & 5.0 & 2.06 & 0.19 & 1.5 & 4.0 & 21.2 \\
\hline 1845.02 & 5.058 & 21.00 & $\ldots$ & $\ldots$ & $\ldots$ & $\ldots$ & 21.1 & 56.2 & 297.4 \\
\hline 1880.01 & 1.151 & 1.49 & 0.52 & 3.7 & 1.70 & 0.18 & 1.5 & 2.8 & 15.6 \\
\hline 1884.01 & 23.120 & 5.00 & 0.92 & 3.6 & 0.95 & 0.55 & 5.1 & 16.3 & 29.7 \\
\hline 1884.02 & 4.775 & 2.63 & $\ldots$ & $\ldots$ & $\ldots$ & $\ldots$ & 2.7 & 8.6 & 15.6 \\
\hline 1890.01 & 4.336 & 1.50 & 1.32 & 3.4 & 0.41 & 0.62 & 1.5 & 3.5 & 5.7 \\
\hline 1891.01 & 15.955 & 1.85 & 0.69 & 4.5 & 2.09 & 0.33 & 1.9 & 6.9 & 21.1 \\
\hline 1891.02 & 8.260 & 1.26 & & $\ldots$ & $\ldots$ & & 1.3 & 4.7 & 14.4 \\
\hline 1916.01 & 20.679 & 2.16 & 0.96 & 2.7 & 0.27 & 0.67 & 2.2 & 5.5 & 8.2 \\
\hline 1916.02 & 9.600 & 1.89 & . & . & . & . & 2.0 & 4.8 & 7.2 \\
\hline 1916.03 & 2.025 & 0.92 & $\ldots$ & $\ldots$ & $\ldots$ & & 1.0 & 2.4 & 3.5 \\
\hline 1979.01 & 2.714 & 1.13 & 0.94 & 3.2 & 0.84 & 0.52 & 1.2 & 2.8 & 5.4 \\
\hline 2009.01 & 86.749 & 2.20 & 0.97 & 4.1 & 1.51 & 0.48 & 2.2 & 7.3 & 15.2 \\
\hline 2059.01 & 6.147 & 0.83 & 0.67 & 1.1 & 0.38 & 0.60 & 1.0 & 1.4 & 2.4 \\
\hline 2059.02 & 2.186 & 0.60 & $\ldots$ & $\ldots$ & $\ldots$ & $\ldots$ & 0.7 & 1.0 & 1.7 \\
\hline
\end{tabular}

Table 4

(Continued)

\begin{tabular}{lccccccccr}
\hline \hline KOI & $\begin{array}{c}P^{\mathrm{a}} \\
(\mathrm{d})\end{array}$ & $\begin{array}{c}R_{p}{ }^{\mathrm{a}} \\
\left(R_{\oplus}\right)\end{array}$ & $\begin{array}{c}R_{\star}{ }^{\mathrm{a}} \\
\left(R_{\odot}\right)\end{array}$ & $\begin{array}{c}\Delta m \\
(\mathrm{mag})\end{array}$ & $\begin{array}{c}\text { sep } \\
\left({ }^{\prime \prime}\right)\end{array}$ & $\begin{array}{c}R_{\star, B}{ }^{\mathrm{b}} \\
\left(R_{\odot}\right)\end{array}$ & $\begin{array}{c}R_{p, A}{ }^{\mathrm{c}} \\
\left(R_{\oplus}\right)\end{array}$ & $\begin{array}{c}R_{p, B} \\
\left(R_{\oplus}\right)\end{array}$ & $\begin{array}{r}R_{p, B_{b g} \mathrm{~d}} \\
\left(R_{\oplus}\right)\end{array}$ \\
\hline 2143.01 & 4.790 & 1.14 & 0.81 & 3.5 & 2.16 & 0.54 & 1.2 & 3.9 & 7.2 \\
2159.01 & 7.597 & 1.07 & 0.88 & 4.0 & 2.00 & 0.48 & 1.1 & 3.8 & 7.7 \\
2159.02 & 2.393 & 0.99 & $\ldots$ & $\ldots$ & $\ldots$ & $\ldots$ & 1.0 & 3.5 & 7.2 \\
2413.01 & 12.905 & 1.32 & 0.65 & 2.1 & 0.31 & 0.46 & 1.4 & 2.7 & 5.8 \\
2413.02 & 31.200 & 1.26 & $\ldots$ & $\ldots$ & $\ldots$ & $\ldots$ & 1.3 & 2.6 & 5.5 \\
2443.01 & 6.792 & 1.20 & 1.09 & 5.4 & 1.39 & 0.41 & 1.2 & 5.3 & 13.1 \\
2443.02 & 11.837 & 1.02 & $\ldots$ & $\ldots$ & $\ldots$ & $\ldots$ & 1.0 & 4.5 & 11.1 \\
2463.01 & 7.467 & 1.02 & 0.97 & 0.8 & 0.62 & 0.94 & 1.2 & 1.7 & 1.8 \\
2486.01 & 4.268 & 2.71 & 1.17 & 0.5 & 0.24 & 1.08 & 3.5 & 4.0 & 3.7 \\
2641.01 & 3.556 & 1.20 & 1.10 & 2.6 & 1.42 & 0.66 & 1.3 & 2.5 & 3.7 \\
2657.01 & 5.224 & 0.60 & 0.80 & 0.3 & 0.73 & 0.89 & 0.8 & 1.0 & 1.1 \\
\hline
\end{tabular}

Notes.

a Values taken from the NASA Exoplanet Archive.

${ }^{b}$ Estimated radius of the stellar companion in the scenario where it is physically bound to the target star. Estimate made according to the absolute magnitude difference in the Kepler band, according the Dartmouth stellar models (Dotter et al. 2008).

${ }^{\mathrm{c}}$ Eclipsing object radius in the scenario where the companion star is the eclipsed object and is physically bound to the target star, assuming the stellar radius of star B as estimated in this table.

${ }^{\mathrm{d}}$ Eclipsing object radius in the scenario where the companion star is the eclipsed object and is a chance-aligned background star with radius $1 R_{\odot}$. We note that a background or foreground object is perhaps unlikely to be solar-type, but this quantification allows for simple scaling of the implied eclipsing object radius.

properties listed in the NASA Exoplanet Archive. For the mass and age of the primary, we use the Dartmouth isochrones to find an absolute magnitude in the observed band (approximating the LP600 bandpass as Kepler band), then we inspect the isochrone to find the mass of a star that is the appropriate amount fainter (according to the observed contrast ratio), and assign the stellar radius $R_{B}$ accordingly.

Table 4 summarizes how the planet radii change under both case $A$ and $B$ for each KOI in all the systems in which we detect companions. We also list an additional case $B_{b g}$ for the situation in which the eclipsed star is not physically bound-since we do not have a constraint on $R_{B}$ in this situation, we simply list the planet radii for the case of $R_{B}=1 R_{\odot}$, which allows for simple scaling.

Interestingly, under case $B$ where the transit is assumed to be around a bound companion, in many cases the implied planet radius is not indicative of a false positive. This is because in order to get a large radius correction there must be a large contrast ratio, which then (in the physically associated scenario) implies that the secondary is a small star, which shrinks the radius correction factor. In fact, the only candidates which attain clearly non-planetary radii under case $B$ are those which already have radii comparable to or larger than Jupiter to begin with. On the other hand, case $B_{b g}$ often suggests a non-planetary radius, as the stellar radius in this case is not bound to shrink as the contrast ratio grows.

We leave a quantitative analysis exploring the relative probability of scenario B being a physically bound or chance-aligned companion to future work. However, we note qualitatively that relatively bright, small-separation companions are more likely to be physically associated, whereas more distant and higher contrast ratio companions are more likely to be foreground/ background objects. 


\subsection{Particularly Interesting Systems}

There are several KOIs with detected companions which we note as being of particular interest, some of which might represent rare false-positive scenarios. Future work will quantitatively assess the true nature of these particular KOIs (e.g., the probability that any given $\mathrm{KOI}$ is a false positive).

\subsubsection{KOI-191: A Probable "Coincident Multiple"}

KOI-191 was identified by Batalha et al. (2013) to have four planet candidates, with periods of approximately $0.7,2.4,15.4$, and 38.7 days. The 15.4 days candidate has an estimated radius of $11 R_{\oplus}$, whereas all the rest are smaller than $1.5 R_{\oplus}$. This system is notable because in the entire current cumulative KOI catalog, there are only four multi-candidate systems that have a planet candidate (either "CANDIDATE" or "NOT DISPOSITIONED" in the NEA) with $10 R_{\oplus}<R<20 R_{\oplus}$ and $P<20 \mathrm{~d}$. Two of these four (KOI-199 and KOI-3627) are marked as two-planet systems but the second candidate in each is identified as a FP in the Q1-Q12 activity table, making them effectively single-candidate systems. The host star of KOI-338 has $R_{\star}=19.2 M_{\odot}$, and its two candidates have radii of 17 and $37 R_{\oplus}$, making that system most likely a stellar multiple system. This leaves KOI-191 as the only multiple-candidate Kepler system including a Jupiter-like candidate with $P<20$ days. By contrast, there are 62 single candidates that match these same radius and period cuts (64 including KOI-199 and KOI-3627).

Based on the apparent rarity of planetary systems with this architecture and the fact that we detect a stellar companion to the KOI-191 host star, we conclude that this is a likely "coincident multiple" system, with KOI-191.01 around one of the stars, and the other three around the other. There are three possibilities: (1) since the companion star (1".69 separation) is $3.1 \mathrm{mag}$ fainter, if it is the host of KOI-191.01, then it is most likely a stellar eclipsing binary; (2) if the primary star hosts .01, then the secondary likely hosts the three-candidate system, in which case .02-.04 are more likely all super-Earth/Neptunesized; (3) it may be the case that all four planets are indeed around the same star, which would make KOI-191 a planetary system of unusual architecture, inviting further study.

\subsubsection{KOI-268: Habitable Zone Candidate?}

KOI-268 hosts a planet candidate in a 110 day orbit. The candidate has a radius of $1.7 R_{\oplus}$ and an equilibrium temperature of $295 \mathrm{~K}$, according to the NEA. However, Robo-AO detects a stellar companion 3.8 mag fainter at a separation of 1".81. We also note the presence of a possible fainter companion at a 2 '.45 separation, a position angle of $306^{\circ}$ and a contrast ratio of $\approx 5.5 \mathrm{mag}$. The equilibrium temperature calculation of the candidate is based on the estimated effective temperature of the host star and the planet is therefore unlikely to be in the habitable zone if it is around one of the companions.

\subsubsection{KOI-628: Possible Triple-system}

KOI-628 has a previously detected faint companion at a separation of 1".83 (Barrado et al. 2013; Lillo-Box et al. 2012). We also re-detect a further possible companion just beyond our detection-target radius, at 2.55 separation.

\subsubsection{KOI-1151: Another Possible Coincident Multiple}

KOI-1151, discovered by this survey to have a companion with $\Delta i \approx 3.5$ at a separation of $0 ! 75$, is another system with unusual architecture that might be best explained if the candidates were shared between the two stars. This system has five detected planet candidates, with periods of 5.25, 7.41, 10.44, 17.45 , and 21.72 days. ${ }^{10}$ What makes this system appear unusual is the presence of the 7.41 days candidate in between the 5.25 days and 10.44 days candidates, which have nearly exact 2:1 commensurability. Of the 22 multi-KOI systems that have a pair of planets within $2 \%$ of exact 2:1 commensurability, only KOI-1151 and KOI-2038 have another candidate between the pair (the inner two planets in this system have been confirmed via transit timing variations by Ming et al. 2013). Migration can tend to deposit planets in or near resonant configurations, but it appears to be unusual for a planet to be stuck between two other planets that are near a strong resonance-perhaps this is an indication that the KOI-1151 system is not a single planetary system at all, but rather two separate systems. Another plausible configuration is that KOIs 1151.02 (the interloper at 7.41 days) and 1151.05 (the 21.72 days candidate) are separated from the other three as those two are near 3:1 commensurability.

\subsubsection{KOI-1442: Largest Contrast Ratio Companion}

We detect a likely companion to KOI-1442 (Kepler magnitude of 12.52) at a separation of 2".24 and a contrast ratio of $\sim 6.7 \mathrm{mag}$. Because of the relatively large separation and large contrast ratio, this detection is more likely to be a background object rather than a physically bound companion. KOI-1442.01 is a planet candidate with a period of 0.67 days and a radius of $1.2 R_{\oplus}$; however, if the fainter companion star is the source of the transit, the radius of the eclipsing object would be significantly larger $-\sim 20 \times$ larger if the companion has the same radius as KOI-1442. Especially since there are hints that very short-period systems may be more likely to be blended binaries (Colón et al. 2012), there might be concern that this candidate is a background eclipsing binary false positive. However, against this hypothesis stands the centroid offset analysis of Bryson et al. (2013) as presented on the NEA, which suggests that the source of the transit could be at most maybe 0.' 5 away from the target position. Therefore, while this system is notable due to the faintness of its detected companion, the companion is unlikely to be the source of a false positive due to its large separation.

5.2.6. KOI-1845: One Likely False Positive in a Two-candidate System

KOI-1845 hosts two planetary candidates: .01 is a $1.5 R_{\oplus}$ candidate in a $1.97-\mathrm{d}$ orbit, and .02 is a $21 R_{\oplus}$ candidate in a 5.06 day orbit. Without any AO observations this system would be suspicious because close-in giant planets are very unlikely to have other planets nearby (see Section 5.2.1); in addition, candidate .02 has a very large Kepler-estimated radius and appears to have a significantly $V$-shaped transit. In this survey we detect a companion 5.0 mag fainter at a separation of 2.06 , and suggest that the most likely explanation for KOI-1845.02 is that this companion is a background eclipsing binary.

\subsubsection{Systems with Secure Small Planets}

There are five systems that host planet candidates with $R_{p}<2 R_{\oplus}$ in which we have detected stellar companions but whose interpretation as small planets $\left(<2 R_{\oplus}\right)$ is nonetheless secure, as long as the companions are physically bound. This

\footnotetext{
10 The NEA cumulative KOI table gives KOI-1151.01 a 5.22 day period rather than 10.44 days, which would be clearly unphysical in the presence of another candidate with a 5.25 day period; the Q1-Q12 table corrects the period of 1151.01-10.44.
} 


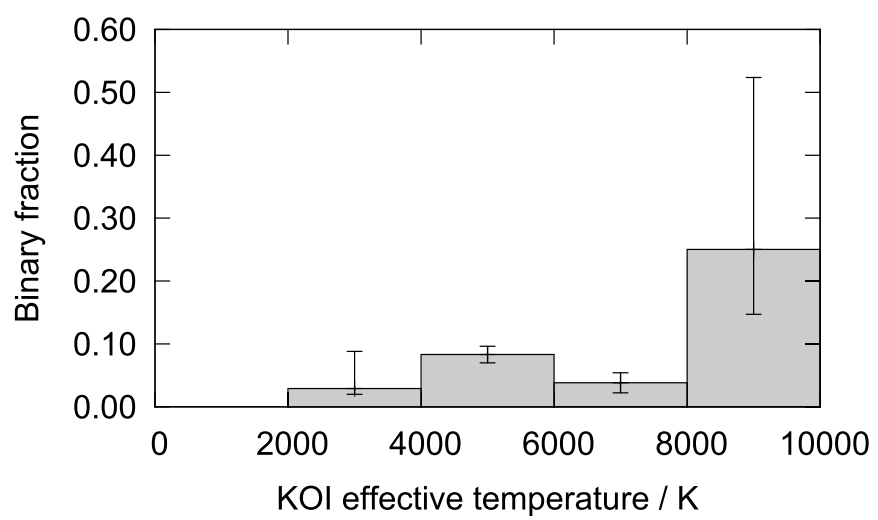

Figure 8. Fraction of KOIs with detected nearby stars as a function of stellar effective temperature.

happens when the candidates are small and the companion is of comparable brightness such that the potential effect of dilution is minimized, even if the eclipse is around the fainter star. The specifics of these systems can be seen in Table 4 but we call attention to them here: KOI-1613, KOI-1619, KOI-2059, KOI-2463, and KOI-2657.

\subsection{Stellar Multiplicity and Kepler Planet Candidates}

Our detection of 53 planetary candidates with nearby stars, from 715 targets, implies an overall nearby-star probability of $7.4 \% \pm 1.0 \%$, within the detectable separation range of our survey $\left(0^{\prime \prime} 15-22^{\prime \prime} .5, \Delta m \lesssim 6\right)$.

In this section we go on to search for broad-scale correlations between stellar multiplicity and planetary candidate properties. The companions we detect may not be physically bound, nor are we sensitive to binaries in all possible orbital locations around these KOIs. This multiplicity rate, therefore, should not be expected give a full description of the physical stellar multiplicity of Kepler planet candidates; however, we can use the current survey results to compare the multiplicity rates of different populations of planet candidates. Future papers from the ongoing Robo-AO survey will investigate the multiplicity properties of Kepler candidates in more detail, including quantifying the effects of association probability and incompleteness.

The above nearby-star probability calculation and the following sections use the binomial distribution to calculate the uncertainty ranges in the multiplicity fractions (e.g., Burgasser et al. 2003) and Fisher exact tests (e.g., Feigelson \& Jogesh Babu 2012) to evaluate the significance of differences in multiplicity between different populations.

\subsubsection{Stellar Multiplicity Rates versus Host-star Temperature}

Figure 8 shows the fraction of multiple stellar systems around Kepler-detected planetary systems as a function of stellar temperature from the Kepler Input Catalog (Brown et al. 2011). The hottest stars appear to have an increased stellar multiplicity fraction, but there is a $16 \%$ probability this is due to chance. We thus do not detect any significant change in the stellar multiplicity fraction with KOI temperature, although the initial survey presented here does not yet cover the entire Kepler sample of non-solar-type stars.

\subsubsection{Stellar Multiplicity and Multiple-planet Systems}

It is expected that multiple-planet systems detected by Kepler are less likely to be false positives than single-planet systems

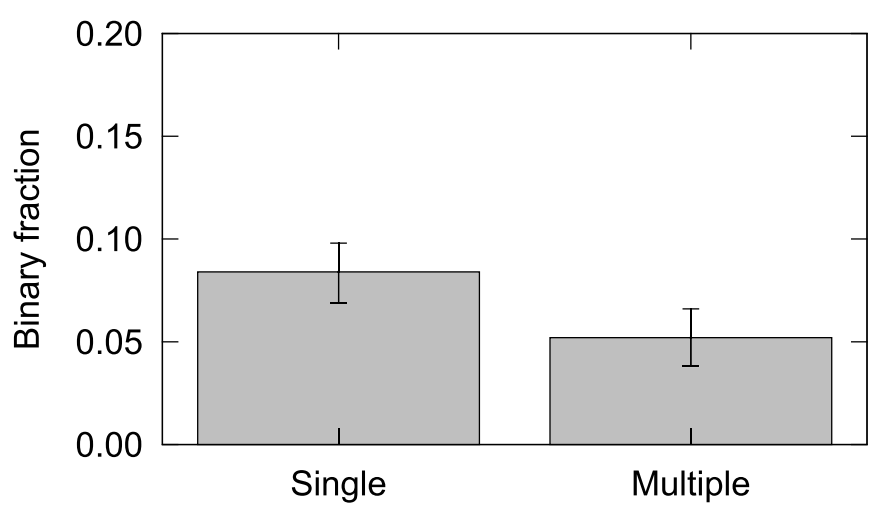

Figure 9. Binarity fractions of KOIs hosting single- and multiple-detected planetary systems.

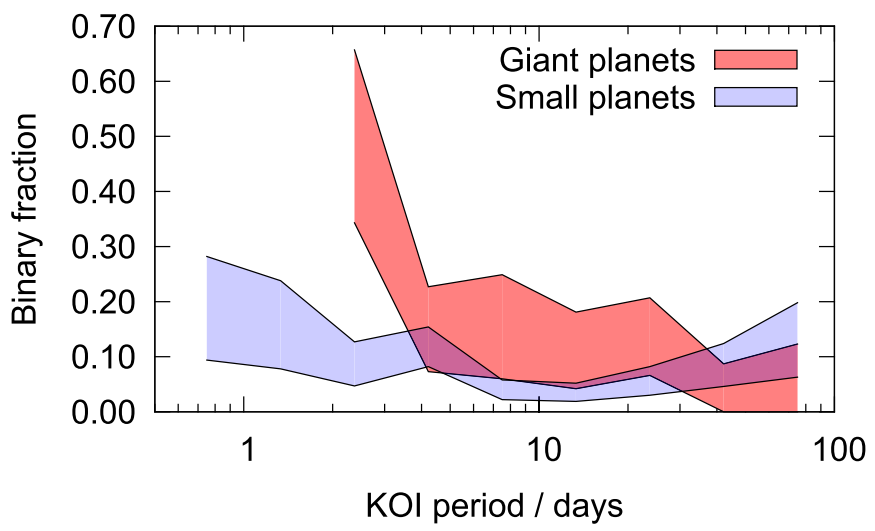

Figure 10. $1 \sigma$ uncertainty regions for binarity fraction as a function of KOI period for two different planetary populations (we split "small" from "giant" at Neptune's radius $\left(3.9 R_{\oplus}\right)$, but the exact value of the split does not significantly affect the uncertainty region shape). The gas giants cut off for shorter periods because of insufficient targets for acceptable statistics.

(A color version of this figure is available in the online journal.)

because there are far fewer false-positive scenarios which can lead to multiple-period false positives. In Figure 9 we show the stellar multiplicity rates for single and multiple planet detections. There is a difference in stellar multiplicity between the single and multiple planet detections, but a Fisher exact test shows a $13 \%$ probability of this being a chance difference due to small-number statistics. At least in the current data set we cannot distinguish stellar multiplicity between single and multiple planet systems.

\subsubsection{Stellar Multiplicity and Close-in Planets}

Stellar binarity has been hypothesized to be important in shaping the architectures of planetary systems, both by regulating planet formation and by dynamically sculpting planets final orbits, such as forcing Kozai oscillations that cause planet migration (Fabrycky \& Tremaine 2007; Katz et al. 2011; Naoz et al. 2012) or by tilting the circumstellar disk (Batygin 2012). If planetary migration is induced by a third body, one would expect to find a correlation between the presence of a detected third body and the presence of short-period planets.

Figure 10 shows the fraction of Kepler planet candidates with nearby stars as a function of the period of the closest-in planet, grouping the planets into two different size ranges. From these raw binarity fractions, where we have not accounted for the probability of physical association, it appears that while small planets do not show a significant change in third-body probability with the orbital period of the Kepler candidate, 


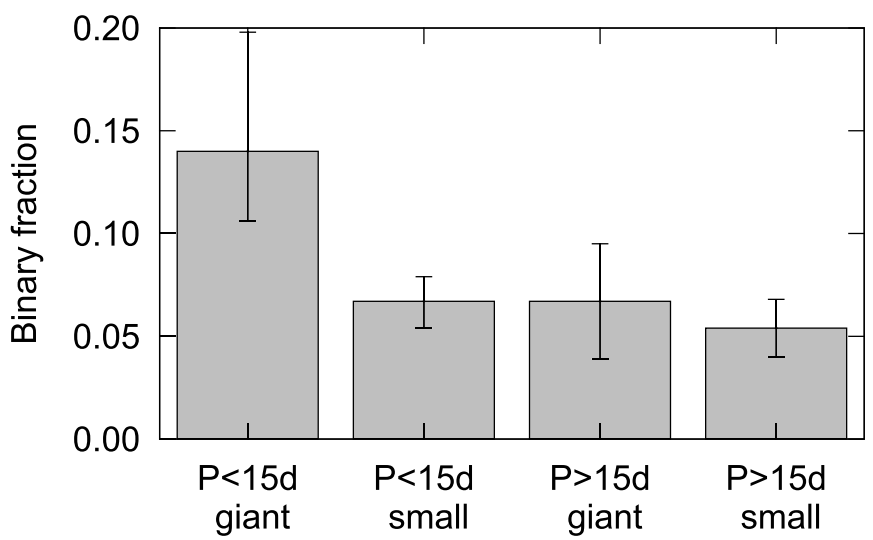

Figure 11. Fraction of KOIs with nearby stars for four different planetary populations. Giant here is shorthand for a radius equal to or larger than that of Neptune. We assign KOIs to these populations if any planet in the system meets the requirements; a small number of multiple-planet systems are therefore assigned to multiple populations.

giant planets show a significant increase at periods less than $\sim 15$ days. Binning all our targets into only four population groups allows us to search for smaller changes in the binarity statistics (Figure 11). We arbitrarily split "small" planets from "giant" planets at Neptune's radius $\left(3.9 R_{\oplus}\right)$, but the exact value of the split does not significantly affect the results; only two of the detected systems have planetary radii within $20 \%$ of the cutoff value. We see that small planets at short periods share the same binarity fraction as all sizes of planets with $>15$ days periods (within statistical errors). However, the shortperiod giant planets again show a significantly increased binarity fraction. A Fisher exact test rejects the hypothesis that the two planetary populations have the same binarity fraction, at the 95\% level.

We can attempt to remove the background asterisms by selecting on the basis of magnitude ratio, as faint background stars are more likely to be chance alignments than roughly equal-brightness companions. Our survey displayed an excess of close-separation bright companions: there are 13 companions with $\Delta m<2$ with separations $<1^{\prime \prime} .5$, and only one at larger radii (Figure 6), while the numbers of fainter companions do not show such a bias. We suggest that this excess reveals a bright-companion population which is more likely to be physically associated than an average companion in the survey.

Selecting the companions with $\Delta m<2$ and separation $<1^{\prime \prime} .5$ leads an increased difference in stellar multiplicity between the planetary populations (Figure 12), increasing the significance to $98 \%$. This approach does not fully account for the probability of each companion being physically associated, and so its results should be interpreted with caution. For example, closein companions are less likely to be rejected by the Kepler centroid-based false-positive tests, but it is not obvious why this rejection would be different for planetary systems with short-period ( $<15$ days) and longer-period KOIs (with a median period of 54 days for the KOIs we surveyed). In fact, the shorterperiod systems have more eclipse events in the Kepler data set and it should therefore be easier to detect a small centroid shift from close-in companions.

On the basis of our current analysis, we suggest that the difference of multiplicity rates between the planetary populations may be tentative evidence for third bodies in stellar systems producing an excess of close-in giant planets. We expect the full Robo-AO surveys to be able to evaluate this possibility at more than the $3 \sigma$ confidence level.

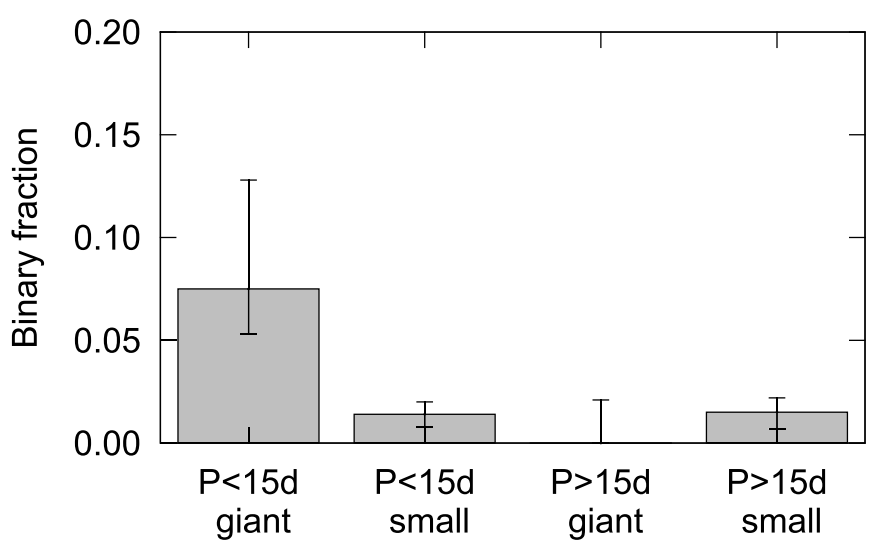

Figure 12. Fraction of KOIs with nearby stars for four different planetary populations - as Figure 11 with only companions with $\Delta m<2$ and separations $<1^{\prime \prime} .5$, removing faint nearby stars which are less likely to be physically associated (we did not detect any bright companions around the 84 longerperiod giant planet KOIs in our survey, so we only show an upper limit). There is a $98 \%$ confidence detection of a difference in stellar multiplicity rates for close-in giant planets compared to further-out giants.

\section{CONCLUSIONS}

We observed 715 Kepler planetary system candidates with the Robo-AO robotic laser adaptive optics system. Our detection of 53 planetary candidates with nearby stars from 715 targets implies an overall nearby-star probability of $7.4 \% \pm 1.0 \%$ at separations between $0^{\prime \prime} .1$ and $2^{\prime \prime} .5$ and $\Delta m \lesssim 6$. We have detailed the effects of the detected nearby stars on the interpretation of the Kepler planetary candidates, including the detection of probable "co-incident" multiples (KOI-191 and KOI1151), multiple-planet systems likely containing false positives (KOI-1845), and the confirmation of five KOIs as roughly Earthradius planets in multiple stellar systems (KOI-1613, KOI-1619, KOI-2059, KOI-2463, and KOI 2657). We have also found tentative, $98 \%$ confidence, evidence for stellar third bodies leading to a $2-3 \times$ increased rate of close-in giant planets.

We expect the ongoing Robo-AO surveys to complete observations of every Kepler planet candidate by the end of 2014 . The increased survey numbers will allow us to search for stellar multiplicity correlations only in multiple-detected planet systems, which are expected to have a much lower false-positive probability, and thus will improve our ability to disentangle false positives from astrophysical effects. The number of multiple systems in our current sample is not large enough to verify our tentative conclusions on the effects of stellar multiplicity on short-period giant planets (in particular, we have only covered one multiple-planet system with a short-period giant planet), but we plan to investigate these possibilities in future data releases. We are also continuing observations of our detected companions to search for common-proper-motion pairs. The completed Robo-AO survey will also allow us to confirm many more Kepler planet candidates and likely find more exotic planetary systems.

We thank the anonymous referee for careful analysis and useful comments on the manuscript. The Robo-AO system is supported by collaborating partner institutions, the California Institute of Technology and the Inter-University Centre for Astronomy and Astrophysics, and by the National Science Foundation under grant Nos. AST-0906060 and AST-0960343, by the Mount Cuba Astronomical Foundation, by a gift from Samuel Oschin. We are grateful to the Palomar Observatory staff for their ongoing support of Robo-AO on the 60 inch telescope, 
Table 5

Full Robo-AO Observation List

\begin{tabular}{|c|c|c|c|c|c|}
\hline KOI & $\mathrm{m}_{\mathrm{i}} / \mathrm{mags}$ & ObsID & Filter & Obs. qual. & Companion? \\
\hline K00001.01 & 11.168 & 2012 Jul 16 & $i$ & high & yes \\
\hline K00002.01 & & $2012 \mathrm{Jul} 16$ & $i$ & high & \\
\hline K00003.01 & & 2012 Jul 16 & $i$ & high & \\
\hline K00005.01 & 11.485 & 2012 Jul 16 & $i$ & high & \\
\hline K00007.01 & 12.038 & 2012 Jul 16 & $i$ & high & \\
\hline K00010.01 & 13.424 & 2012 Jul 16 & $i$ & medium & \\
\hline K00012.01 & 11.245 & 2012 Jul 17 & $i$ & high & \\
\hline K00013.01 & 10.548 & 2012 Oct 6 & $i$ & high & yes \\
\hline K00017.01 & 13.094 & 2012 Jul 16 & $i$ & medium & \\
\hline K00018.01 & 13.148 & 2012 Jul 16 & $i$ & medium & \\
\hline K00022.01 & 13.265 & 2012 Jul 16 & $i$ & medium & \\
\hline K00041.01 & 11.03 & 2012 Jul 16 & $i$ & high & \\
\hline K00044.01 & 13.268 & 2012 Jul 16 & $i$ & low & \\
\hline K00046.01 & 13.497 & 2012 Jul 16 & $i$ & medium & \\
\hline K00049.01 & 13.508 & 2012 Jul 16 & $i$ & low & \\
\hline K00063.01 & 11.379 & 2012 Jul 16 & $i$ & high & \\
\hline K00064.01 & 12.866 & 2012 Jul 16 & $i$ & medium & \\
\hline K00069.01 & 9.739 & 2012 Jul 16 & $i$ & high & \\
\hline K00070.01 & 12.284 & 2012 Jul 16 & $i$ & medium & \\
\hline K00075.01 & 10.617 & 2012 Jul 16 & $i$ & high & \\
\hline K00082.01 & 11.15 & 2012 Jul 16 & $i$ & high & \\
\hline K00084.01 & 11.694 & 2012 Jul 16 & $i$ & high & \\
\hline K00085.01 & 10.882 & 2012 Jul 16 & $i$ & high & \\
\hline K00087.01 & 11.478 & 2012 Jul 16 & $i$ & high & \\
\hline K00089.01 & 11.649 & 2012 Jul 16 & $i$ & medium & \\
\hline K00092.01 & 11.506 & 2012 Jul 16 & $i$ & high & \\
\hline K00094.01 & 12.057 & 2012 Jul 16 & $i$ & medium & \\
\hline K00097.01 & 12.724 & 2012 Jul 17 & $i$ & medium & yes \\
\hline K00098.01 & 12.024 & 2012 Jul 17 & $i$ & high & yes \\
\hline K00099.01 & 12.68 & $2012 \mathrm{Jul} 16$ & $i$ & medium & \\
\hline K00100.01 & 12.466 & $2012 \mathrm{Jul} 16$ & $i$ & medium & \\
\hline K00102.01 & 12.384 & 2012 Jul 16 & $i$ & medium & \\
\hline K00103.01 & 12.399 & 2012 Jul 16 & $i$ & medium & \\
\hline K00105.01 & 12.649 & 2012 Jul 16 & $i$ & medium & \\
\hline K00107.01 & 12.53 & 2012 Jul 16 & $i$ & medium & \\
\hline K00108.01 & 12.132 & $2012 \mathrm{Jul} 16$ & $i$ & high & \\
\hline K00110.01 & 12.545 & 2012 Jul 16 & $i$ & medium & \\
\hline K00111.01 & 12.442 & 2012 Jul 16 & $i$ & medium & \\
\hline K00112.01 & 12.602 & 2012 Jul 18 & $i$ & medium & \\
\hline K00113.01 & 12.163 & 2012 Jul 17 & $i$ & high & \\
\hline K00115.01 & 12.654 & 2012 Jul 16 & $i$ & medium & \\
\hline K00117.01 & 12.309 & 2012 Jul 16 & $i$ & medium & \\
\hline K00118.01 & 12.195 & 2012 Jul 17 & $i$ & medium & \\
\hline K00119.01 & 12.452 & 2012 Jul 16 & $i$ & low & yes \\
\hline K00122.01 & 12.161 & 2012 Jul 16 & $i$ & medium & \\
\hline K00124.01 & 12.784 & 2012 Jul 16 & $i$ & low & \\
\hline K00128.01 & 13.54 & 2012 Jul 16 & $i$ & low & \\
\hline K00131.01 & 13.64 & 2012 Jul 16 & $i$ & low & \\
\hline K00137.01 & 13.287 & 2012 Jul 17 & $i$ & low & \\
\hline K00139.01 & 13.327 & 2012 Jul 17 & $i$ & low & \\
\hline K00141.01 & 13.441 & 2012 Jul 18 & $i$ & medium & yes \\
\hline K00142.01 & 12.895 & 2012 Jul 17 & $i$ & low & \\
\hline K00144.01 & 13.329 & 2012 Jul 17 & $i$ & low & \\
\hline K00148.01 & 12.761 & 2012 Jul 17 & $i$ & low & \\
\hline K00149.01 & 13.167 & 2012 Jul 17 & $i$ & low & \\
\hline K00152.01 & 13.761 & 2012 Jul 17 & $i$ & low & \\
\hline K00153.01 & 13.097 & 2012 Jul 17 & LP600 & medium & \\
\hline K00156.01 & 13.334 & 2012 Sep 1 & LP600 & high & \\
\hline K00157.01 & 13.508 & 2012 Sep 1 & LP600 & high & \\
\hline K00159.01 & 13.243 & 2012 Jul 18 & LP600 & high & \\
\hline K00161.01 & 12.99 & 2012 Jul 18 & LP600 & high & \\
\hline K00162.01 & 13.626 & 2012 Jul 18 & LP600 & medium & yes \\
\hline K00165.01 & 13.665 & 2012 Jul 17 & LP600 & medium & \\
\hline K00166.01 & 13.315 & 2012 Jul 17 & LP600 & medium & \\
\hline K00167.01 & 13.15 & 2012 Jul 17 & LP600 & high & \\
\hline
\end{tabular}

Table 5

(Continued)

\begin{tabular}{|c|c|c|c|c|c|}
\hline KOI & $\mathrm{m}_{\mathrm{i}} / \mathrm{mags}$ & ObsID & Filter & Obs. qual. & Companion? \\
\hline K00168.01 & 13.244 & 2012 Jul 17 & LP600 & high & \\
\hline K00171.01 & 13.575 & 2012 Jul 17 & LP600 & high & \\
\hline K00172.01 & 13.559 & 2012 Jul 18 & LP600 & high & \\
\hline K00173.01 & 13.659 & 2012 Jul 18 & LP600 & high & \\
\hline K00174.01 & 13.449 & 2012 Jul 18 & LP600 & high & yes \\
\hline K00176.01 & 13.307 & 2012 Jul 18 & LP600 & high & \\
\hline K00177.01 & 12.979 & 2012 Jul 18 & $i$ & medium & yes \\
\hline K00179.01 & 13.765 & 2012 Jul 18 & LP600 & high & \\
\hline K00180.01 & 12.813 & 2012 Jul 18 & $i$ & medium & \\
\hline K00191.01 & 14.747 & 2012 Sep 1 & LP600 & low & yes \\
\hline K00197.01 & 13.706 & 2012 Jul 18 & $i$ & medium & \\
\hline K00201.01 & 13.785 & 2012 Jul 18 & LP600 & high & \\
\hline K00203.01 & 13.928 & 2012 Jul 18 & LP600 & high & \\
\hline K00209.01 & 14.131 & 2012 Sep 1 & LP600 & medium & \\
\hline K00211.01 & 14.82 & 2012 Sep 14 & LP600 & low & \\
\hline K00214.01 & 14.003 & 2012 Jul 18 & LP600 & high & \\
\hline K00216.01 & 14.4 & 2012 Sep 1 & LP600 & low & \\
\hline K00219.01 & 13.925 & 2012 Jul 18 & LP600 & high & \\
\hline K00220.01 & 14.011 & 2012 Sep 1 & LP600 & medium & \\
\hline K00222.01 & 14.315 & 2012 Sep 1 & LP600 & low & \\
\hline K00223.01 & 14.447 & 2012 Sep 1 & LP600 & medium & \\
\hline K00232.01 & 14.067 & 2012 Jul 18 & LP600 & medium & \\
\hline K00237.01 & 13.964 & 2012 Jul 18 & LP600 & medium & \\
\hline K00238.01 & 13.891 & 2012 Sep 1 & LP600 & medium & \\
\hline K00241.01 & 13.881 & 2012 Jul 18 & LP600 & medium & \\
\hline K00244.01 & & 2012 Jul 18 & $i$ & high & \\
\hline K00246.01 & 9.82 & 2012 Jul 18 & $i$ & high & \\
\hline K00247.01 & 13.585 & 2012 Aug 3 & LP600 & high & \\
\hline K00248.01 & 14.68 & 2012 Aug 3 & LP600 & medium & \\
\hline K00250.01 & 14.887 & 2012 Aug 3 & LP600 & low & \\
\hline K00253.01 & 14.667 & 2012 Aug 3 & LP600 & medium & \\
\hline K00254.01 & 15.364 & 2012 Aug 3 & LP600 & low & \\
\hline K00256.01 & 14.636 & 2012 Jul 15 & LP600 & medium & \\
\hline K00260.01 & & 2012 Jul 18 & $i$ & high & \\
\hline K00261.01 & 10.109 & 2012 Jul 18 & $i$ & high & \\
\hline K00263.01 & 10.647 & 2012 Jul 18 & $i$ & high & \\
\hline K00268.01 & & 2012 Sep 14 & LP600 & high & yes \\
\hline K00269.01 & 10.823 & 2012 Jul 18 & $i$ & high & \\
\hline K00270.01 & & 2012 Jul 17 & $i$ & high & \\
\hline K00273.01 & 11.262 & 2012 Jul 18 & $i$ & high & \\
\hline K00275.01 & & 2012 Jul 18 & $i$ & high & \\
\hline K00276.01 & 11.711 & 2012 Jul 18 & $i$ & high & \\
\hline K00277.01 & & 2012 Jul 18 & $i$ & high & \\
\hline K00279.01 & 11.563 & 2012 Jul 18 & $i$ & medium & \\
\hline K00281.01 & 11.77 & 2012 Jul 18 & $i$ & high & \\
\hline K00282.01 & & 2012 Jul 18 & $i$ & high & \\
\hline K00283.01 & 11.334 & 2012 Jul 18 & $i$ & high & \\
\hline K00288.01 & & 2012 Jul 18 & $i$ & high & \\
\hline K00291.01 & 12.642 & 2012 Jul 18 & $i$ & high & \\
\hline K00294.01 & 12.511 & 2012 Jul 18 & $i$ & high & \\
\hline K00296.01 & 12.77 & 2012 Aug 2 & $i$ & medium & \\
\hline K00297.01 & 12.042 & 2012 Aug 2 & $i$ & high & \\
\hline K00299.01 & 12.675 & 2012 Jul 18 & $i$ & medium & \\
\hline K00301.01 & 12.586 & 2012 Jul 18 & $i$ & high & \\
\hline K00302.01 & 11.969 & 2012 Jul 18 & $i$ & high & \\
\hline K00303.01 & 11.994 & 2012 Jul 18 & $i$ & high & \\
\hline K00305.01 & 12.606 & 2012 Jul 18 & $i$ & medium & \\
\hline K00306.01 & 12.363 & 2012 Jul 18 & $i$ & low & yes \\
\hline K00307.01 & 12.65 & 2012 Aug 2 & $i$ & medium & \\
\hline K00308.01 & 12.205 & 2012 Jul 18 & $i$ & medium & \\
\hline K00312.01 & & 2012 Jul 28 & $i$ & high & \\
\hline K00313.01 & 12.736 & 2012 Aug 2 & $i$ & high & \\
\hline K00314.01 & 12.457 & 2012 Aug 3 & LP600 & high & \\
\hline K00315.01 & 12.63 & 2012 Jul 28 & $i$ & medium & \\
\hline K00316.01 & 12.494 & 2012 Aug 2 & $i$ & high & \\
\hline
\end{tabular}


Table 5

(Continued)

\begin{tabular}{|c|c|c|c|c|c|}
\hline KOI & $\mathrm{m}_{\mathrm{i}} / \mathrm{mags}$ & ObsID & Filter & Obs. qual. & Companion? \\
\hline K00317.01 & 12.751 & 2012 Jul 28 & $i$ & medium & \\
\hline K00319.01 & & 2012 Aug 2 & $i$ & high & \\
\hline K00321.01 & 12.312 & 2012 Aug 2 & $i$ & high & \\
\hline K00323.01 & 12.24 & 2012 Aug 2 & $i$ & high & \\
\hline K00327.01 & 12.858 & 2012 Aug 2 & $i$ & medium & \\
\hline K00330.01 & 13.73 & 2012 Jul 28 & LP600 & high & \\
\hline K00331.01 & 13.277 & 2012 Jul 28 & $i$ & medium & \\
\hline K00332.01 & 12.847 & 2012 Jul 28 & $i$ & high & \\
\hline K00333.01 & 13.265 & 2012 Aug 2 & $i$ & medium & \\
\hline K00337.01 & 13.746 & 2012 Aug 2 & LP600 & medium & \\
\hline K00339.01 & 13.616 & 2012 Aug 2 & LP600 & medium & \\
\hline K00340.01 & 12.82 & 2012 Jul 28 & $i$ & medium & \\
\hline K00341.01 & 13.106 & 2012 Jul 28 & $i$ & medium & \\
\hline K00343.01 & 13.013 & 2012 Aug 2 & $i$ & medium & \\
\hline K00344.01 & 13.211 & 2012 Aug 2 & $i$ & medium & \\
\hline K00345.01 & 13.005 & 2012 Aug 2 & $i$ & medium & \\
\hline K00348.01 & 13.555 & 2012 Aug 3 & LP600 & high & \\
\hline K00349.01 & 13.382 & 2012 Aug 2 & LP600 & high & \\
\hline K00350.01 & 13.202 & 2012 Aug 2 & $i$ & medium & \\
\hline K00352.01 & 13.579 & 2012 Jul 28 & LP600 & high & \\
\hline K00353.01 & 13.251 & 2012 Aug 2 & $i$ & low & \\
\hline K00356.01 & 13.532 & 2012 Jul 28 & LP600 & high & yes \\
\hline K00360.01 & 12.823 & 2012 Aug 2 & $i$ & medium & \\
\hline K00361.01 & 12.914 & 2012 Aug 2 & $i$ & medium & \\
\hline K00365.01 & 10.992 & 2012 Jul 28 & $i$ & high & \\
\hline K00366.01 & & 2012 Aug 2 & $i$ & high & \\
\hline K00368.01 & 11.598 & 2012 Aug 2 & $i$ & high & \\
\hline K00371.01 & 11.895 & 2012 Jul 28 & $i$ & high & \\
\hline K00372.01 & 12.208 & 2012 Jul 28 & $i$ & high & \\
\hline K00373.01 & 12.593 & 2012 Aug 2 & $i$ & medium & \\
\hline K00377.01 & 13.613 & 2012 Sep 1 & LP600 & medium & \\
\hline K00384.01 & 13.106 & 2012 Aug 2 & $i$ & low & \\
\hline K00385.01 & 13.211 & 2012 Aug 2 & $i$ & medium & \\
\hline K00386.01 & 13.661 & 2012 Aug 2 & $i$ & low & \\
\hline K00388.01 & 13.448 & 2012 Aug 2 & $i$ & medium & \\
\hline K00392.01 & 13.745 & 2012 Aug 5 & LP600 & medium & \\
\hline K00393.01 & 13.395 & 2012 Aug 2 & $i$ & low & \\
\hline K00401.01 & 13.729 & 2012 Aug 5 & LP600 & medium & yes \\
\hline K00403.01 & 13.953 & 2012 Aug 5 & LP600 & medium & \\
\hline K00408.01 & 14.766 & 2012 Sep 1 & LP600 & low & \\
\hline K00409.01 & 13.965 & 2012 Sep 14 & LP600 & medium & \\
\hline K00413.01 & 14.512 & 2012 Sep 1 & LP600 & low & \\
\hline K00415.01 & 13.914 & 2012 Aug 5 & LP600 & medium & \\
\hline K00416.01 & 14.019 & 2012 Sep 1 & LP600 & low & \\
\hline K00427.01 & 14.37 & 2012 Sep 1 & LP600 & low & \\
\hline K00431.01 & 14.004 & 2012 Sep 1 & LP600 & medium & \\
\hline K00435.01 & 14.342 & 2012 Sep 1 & LP600 & low & \\
\hline K00439.01 & 14.063 & 2012 Aug 5 & LP600 & medium & \\
\hline K00440.01 & 13.861 & 2012 Sep 1 & LP600 & medium & \\
\hline K00442.01 & 13.806 & 2012 Aug 5 & LP600 & medium & \\
\hline K00444.01 & 13.909 & 2012 Aug 5 & LP600 & medium & \\
\hline K00456.01 & 14.407 & 2012 Sep 1 & LP600 & medium & \\
\hline K00457.01 & 13.894 & 2012 Sep 1 & LP600 & medium & \\
\hline K00459.01 & 14.028 & 2012 Sep 1 & LP600 & medium & \\
\hline K00463.01 & 13.999 & 2012 Aug 3 & LP600 & medium & \\
\hline K00464.01 & 14.113 & 2012 Sep 1 & LP600 & medium & \\
\hline K00465.01 & 14.017 & 2012 Aug 5 & LP600 & medium & \\
\hline K00471.01 & 14.198 & 2012 Sep 1 & LP600 & medium & \\
\hline K00474.01 & 14.131 & 2012 Sep 1 & LP600 & low & \\
\hline K00478.01 & 13.58 & 2012 Aug 4 & LP600 & high & \\
\hline K00481.01 & 14.446 & 2012 Sep 1 & LP600 & medium & \\
\hline K00486.01 & 13.934 & 2012 Aug 5 & LP600 & medium & \\
\hline K00490.01 & 13.688 & 2012 Aug 5 & LP600 & medium & \\
\hline K00497.01 & 14.423 & 2012 Sep 1 & LP600 & low & \\
\hline K00508.01 & 14.146 & 2012 Sep 1 & LP600 & medium & \\
\hline
\end{tabular}

Table 5

(Continued)

\begin{tabular}{|c|c|c|c|c|c|}
\hline KOI & $\mathrm{m}_{\mathrm{i}} / \mathrm{mags}$ & ObsID & Filter & Obs. qual. & Companion? \\
\hline K00509.01 & 14.638 & 2012 Sep 1 & LP600 & low & \\
\hline K00511.01 & 14.017 & 2012 Sep 1 & LP600 & medium & yes \\
\hline K00517.01 & 13.806 & 2012 Aug 5 & LP600 & high & \\
\hline K00519.01 & 14.737 & 2012 Sep 1 & LP600 & medium & \\
\hline K00520.01 & 14.255 & 2012 Sep 1 & LP600 & medium & \\
\hline K00523.01 & 14.822 & 2012 Sep 1 & LP600 & low & \\
\hline K00528.01 & 14.364 & 2012 Sep 1 & LP600 & low & \\
\hline K00531.01 & 13.849 & 2012 Aug 3 & LP600 & high & \\
\hline K00534.01 & 14.344 & 2012 Sep 1 & LP600 & medium & \\
\hline K00542.01 & 14.12 & 2012 Sep 1 & LP600 & medium & \\
\hline K00543.01 & 14.442 & 2012 Sep 1 & LP600 & low & \\
\hline K00546.01 & 14.717 & 2012 Sep 1 & LP600 & low & \\
\hline K00548.01 & 13.874 & 2012 Aug 5 & LP600 & medium & \\
\hline K00550.01 & 13.869 & 2012 Aug 5 & LP600 & medium & \\
\hline K00551.01 & 14.725 & 2012 Sep 1 & LP600 & low & \\
\hline K00555.01 & 14.499 & 2012 Aug 5 & LP600 & medium & \\
\hline K00561.01 & 13.732 & 2012 Aug 5 & LP600 & medium & \\
\hline K00564.01 & 14.642 & 2012 Sep 2 & LP600 & low & \\
\hline K00567.01 & 14.126 & 2012 Sep 2 & LP600 & medium & \\
\hline K00568.01 & 13.895 & 2012 Aug 5 & LP600 & medium & \\
\hline K00569.01 & 14.172 & 2012 Sep 2 & LP600 & medium & \\
\hline K00571.01 & 14.015 & 2012 Aug 3 & LP600 & high & \\
\hline K00572.01 & 13.96 & 2012 Jul 28 & $i$ & low & \\
\hline K00574.01 & 14.579 & 2012 Sep 2 & LP600 & low & \\
\hline K00579.01 & 13.858 & 2012 Aug 5 & LP600 & medium & \\
\hline K00582.01 & 14.529 & 2012 Sep 2 & LP600 & medium & \\
\hline K00590.01 & 14.444 & 2012 Sep 2 & LP600 & low & \\
\hline K00593.01 & 14.754 & 2012 Sep 2 & LP600 & low & \\
\hline K00597.01 & 14.721 & 2012 Sep 2 & LP600 & low & \\
\hline K00601.01 & 14.515 & 2012 Sep 2 & LP600 & medium & \\
\hline K00611.01 & 13.866 & 2012 Aug 5 & LP600 & medium & \\
\hline K00612.01 & 13.871 & 2012 Aug 5 & LP600 & medium & \\
\hline K00620.01 & 14.467 & 2012 Sep 2 & LP600 & medium & \\
\hline K00623.01 & 11.685 & 2012 Aug 3 & $i$ & high & \\
\hline K00624.01 & 13.39 & 2012 Aug 3 & $i$ & medium & \\
\hline K00625.01 & 13.433 & 2012 Aug 3 & $i$ & medium & \\
\hline K00626.01 & 13.339 & 2012 Aug 3 & $i$ & medium & \\
\hline K00627.01 & 13.119 & 2012 Aug 3 & $i$ & medium & \\
\hline K00628.01 & 13.744 & 2012 Aug 3 & $i$ & medium & yes \\
\hline K00629.01 & 13.788 & 2012 Aug 4 & $i$ & medium & \\
\hline K00632.01 & 13.124 & 2012 Aug 4 & $i$ & medium & \\
\hline K00633.01 & 13.663 & 2012 Aug 4 & $i$ & medium & \\
\hline K00635.01 & 12.88 & 2012 Aug 4 & $i$ & medium & \\
\hline K00638.01 & 13.394 & 2012 Aug 4 & $i$ & medium & \\
\hline K00639.01 & 13.354 & 2012 Jul 28 & $i$ & medium & \\
\hline K00640.01 & 13.058 & 2012 Jul 28 & $i$ & low & yes \\
\hline K00644.01 & 13.474 & 2012 Aug 4 & $i$ & medium & \\
\hline K00647.01 & 13.413 & 2012 Aug 4 & $i$ & medium & \\
\hline K00649.01 & 13.157 & 2012 Aug 4 & $i$ & medium & \\
\hline K00650.01 & 13.293 & 2012 Aug 4 & $i$ & medium & \\
\hline K00654.01 & 13.789 & 2012 Aug 4 & $i$ & medium & \\
\hline K00655.01 & 12.872 & 2012 Aug 4 & $i$ & medium & \\
\hline K00657.01 & 13.517 & 2012 Aug 4 & $i$ & medium & \\
\hline K00658.01 & 13.789 & 2012 Aug 4 & $i$ & medium & \\
\hline K00659.01 & 13.297 & 2012 Aug 4 & $i$ & medium & \\
\hline K00660.01 & 13.283 & 2012 Aug 4 & $i$ & medium & \\
\hline K00661.01 & 13.731 & 2012 Aug 4 & $i$ & medium & \\
\hline K00662.01 & 13.168 & 2012 Aug 4 & $i$ & medium & \\
\hline K00663.01 & 13.016 & 2012 Sep 2 & LP600 & high & \\
\hline K00664.01 & 13.287 & 2012 Aug 4 & $i$ & medium & \\
\hline K00665.01 & 13.005 & 2012 Aug 4 & $i$ & medium & \\
\hline K00666.01 & 13.518 & 2012 Aug 4 & $i$ & medium & \\
\hline K00671.01 & 13.511 & 2012 Aug 4 & $i$ & medium & \\
\hline K00673.01 & 13.211 & 2012 Aug 4 & $i$ & medium & \\
\hline K00674.01 & 13.435 & 2012 Aug 4 & $i$ & medium & \\
\hline
\end{tabular}


Table 5

(Continued)

\begin{tabular}{|c|c|c|c|c|c|}
\hline KOI & $\mathrm{m}_{\mathrm{i}} / \mathrm{mags}$ & ObsID & Filter & Obs. qual. & Companion? \\
\hline K00676.01 & 13.371 & 2012 Sep 2 & LP600 & high & \\
\hline K00679.01 & 13.038 & 2012 Aug 4 & $i$ & medium & \\
\hline K00680.01 & 13.485 & 2012 Aug 4 & $i$ & medium & \\
\hline K00682.01 & 13.692 & 2012 Aug 4 & $i$ & medium & \\
\hline K00684.01 & 13.575 & 2012 Aug 4 & $i$ & medium & \\
\hline K00685.01 & 13.77 & 2012 Aug 4 & $i$ & medium & \\
\hline K00686.01 & 13.346 & 2012 Aug 4 & $i$ & medium & \\
\hline K00687.01 & 13.613 & 2012 Aug 4 & $i$ & medium & yes \\
\hline K00688.01 & 13.849 & 2012 Sep 14 & LP600 & medium & yes \\
\hline K00689.01 & 13.548 & 2012 Aug 4 & $i$ & medium & \\
\hline K00691.01 & 13.803 & 2012 Aug 5 & $i$ & low & \\
\hline K00692.01 & 13.457 & 2012 Aug 5 & $i$ & medium & \\
\hline K00694.01 & 13.741 & 2012 Aug 5 & $i$ & low & \\
\hline K00695.01 & 13.276 & 2012 Aug 5 & $i$ & medium & \\
\hline K00698.01 & 13.52 & 2012 Aug 5 & $i$ & low & \\
\hline K00700.01 & 13.38 & 2012 Aug 5 & $i$ & medium & \\
\hline K00701.01 & 13.429 & 2012 Aug 5 & $i$ & low & \\
\hline K00703.01 & 13.162 & 2012 Aug 5 & $i$ & medium & \\
\hline K00704.01 & 13.46 & 2012 Aug 5 & $i$ & medium & \\
\hline K00707.01 & 13.815 & 2012 Aug 5 & $i$ & low & \\
\hline K00708.01 & 13.837 & 2012 Aug 5 & $i$ & low & \\
\hline K00709.01 & 13.716 & 2012 Aug 5 & $i$ & medium & \\
\hline K00710.01 & 13.128 & 2012 Aug 5 & $i$ & medium & \\
\hline K00711.01 & 13.735 & 2012 Aug 5 & $i$ & medium & \\
\hline K00712.01 & 13.51 & 2012 Aug 5 & $i$ & medium & yes \\
\hline K00714.01 & 13.184 & 2012 Aug 5 & $i$ & medium & \\
\hline K00716.01 & 13.576 & 2012 Aug 5 & $i$ & low & \\
\hline K00717.01 & 13.182 & 2012 Aug 5 & $i$ & medium & \\
\hline K00718.01 & 13.588 & 2012 Aug 5 & $i$ & low & \\
\hline K00719.01 & 12.899 & 2012 Aug 5 & $i$ & medium & \\
\hline K00720.01 & 13.489 & 2012 Aug 5 & $i$ & low & \\
\hline K00721.01 & 13.439 & 2012 Aug 5 & $i$ & low & \\
\hline K00722.01 & 13.343 & 2012 Aug 5 & $i$ & high & \\
\hline K00723.01 & 14.795 & 2012 Sep 2 & LP600 & low & \\
\hline K00738.01 & 15.063 & 2012 Sep 2 & LP600 & low & \\
\hline K00739.01 & 14.931 & 2012 Aug 3 & LP600 & medium & \\
\hline K00756.01 & 15.492 & 2012 Sep 2 & LP600 & low & \\
\hline K00781.01 & 15.267 & 2012 Aug 3 & LP600 & low & \\
\hline K00800.01 & 15.341 & 2012 Sep 2 & LP600 & low & \\
\hline K00817.01 & 14.793 & 2012 Aug 3 & LP600 & medium & \\
\hline K00818.01 & 15.192 & 2012 Aug 4 & LP600 & medium & \\
\hline K00834.01 & 14.862 & 2012 Sep 2 & LP600 & low & \\
\hline K00835.01 & 14.884 & 2012 Sep 2 & LP600 & low & \\
\hline K00837.01 & 15.325 & 2012 Sep 2 & LP600 & low & \\
\hline K00842.01 & 15.001 & 2012 Sep 2 & LP600 & low & \\
\hline K00853.01 & 15.039 & 2012 Sep 2 & LP600 & low & \\
\hline K00854.01 & 15.162 & 2012 Aug 3 & LP600 & low & \\
\hline K00857.01 & 14.787 & 2012 Sep 2 & LP600 & low & \\
\hline K00872.01 & 14.98 & 2012 Sep 2 & LP600 & low & \\
\hline K00874.01 & 14.716 & 2012 Sep 2 & LP600 & low & \\
\hline K00877.01 & 14.547 & 2012 Jun 17 & LP600 & low & \\
\hline K00880.01 & 14.918 & 2012 Sep 2 & LP600 & low & \\
\hline K00884.01 & 14.755 & 2012 Sep 2 & LP600 & low & \\
\hline K00886.01 & 15.175 & 2012 Aug 4 & LP600 & medium & \\
\hline K00896.01 & 14.974 & 2012 Sep 2 & LP600 & low & \\
\hline K00898.01 & 15.221 & 2012 Aug 4 & LP600 & medium & \\
\hline K00899.01 & 14.543 & 2012 Aug 4 & LP600 & medium & \\
\hline K00906.01 & 15.155 & 2012 Sep 2 & LP600 & low & \\
\hline K00907.01 & 14.983 & 2012 Sep 2 & LP600 & low & \\
\hline K00921.01 & 15.229 & 2012 Sep 2 & LP600 & low & \\
\hline K00935.01 & 15.086 & 2012 Sep 2 & LP600 & low & \\
\hline K00936.01 & 14.371 & 2012 Aug 4 & LP600 & medium & \\
\hline K00938.01 & 15.328 & 2012 Sep 2 & LP600 & low & \\
\hline K00939.01 & 14.849 & 2012 Sep 2 & LP600 & low & \\
\hline K00947.01 & 14.564 & 2012 Aug 4 & LP600 & medium & \\
\hline
\end{tabular}

Table 5

(Continued)

\begin{tabular}{|c|c|c|c|c|c|}
\hline KOI & $\mathrm{m}_{\mathrm{i}} / \mathrm{mags}$ & ObsID & Filter & Obs. qual. & Companion? \\
\hline K00975.01 & & 2012 Jul 17 & $i$ & high & \\
\hline K00977.01 & & 2012 Aug 3 & $i$ & high & \\
\hline K00984.01 & 11.353 & 2012 Aug 3 & $i$ & high & yes \\
\hline K00986.01 & 13.908 & 2012 Aug 3 & $i$ & low & \\
\hline K00987.01 & 12.327 & 2012 Aug 3 & $i$ & medium & yes \\
\hline K00988.01 & 13.259 & 2012 Aug 3 & $i$ & medium & \\
\hline K00991.01 & 13.368 & 2012 Aug 3 & $i$ & medium & \\
\hline K01001.01 & 12.851 & 2012 Aug 3 & $i$ & medium & \\
\hline K01002.01 & 13.362 & 2012 Aug 3 & $i$ & medium & yes \\
\hline K01010.01 & 13.463 & 2012 Aug 3 & $i$ & medium & \\
\hline K01015.01 & 14.349 & 2012 Sep 3 & LP600 & medium & \\
\hline K01019.01 & 9.961 & 2012 Aug 3 & $i$ & high & \\
\hline K01020.01 & 12.712 & 2012 Aug 3 & $i$ & medium & \\
\hline K01032.01 & 13.497 & 2012 Aug 3 & $i$ & medium & \\
\hline K01050.01 & 13.696 & 2012 Aug 3 & $i$ & low & yes \\
\hline K01052.01 & 15.201 & 2012 Sep 3 & LP600 & medium & \\
\hline K01054.01 & 11.662 & 2012 Aug 3 & $i$ & high & \\
\hline K01060.01 & 14.221 & 2012 Sep 3 & LP600 & medium & \\
\hline K01070.01 & 15.348 & 2012 Sep 3 & LP600 & low & \\
\hline K01078.01 & 14.846 & 2012 Aug 4 & LP600 & medium & \\
\hline K01085.01 & 14.651 & 2012 Aug 4 & LP600 & medium & \\
\hline K01089.01 & 14.501 & 2012 Sep 3 & LP600 & medium & \\
\hline K01102.01 & 14.711 & 2012 Aug 5 & LP600 & low & \\
\hline K01113.01 & 13.54 & 2012 Aug 5 & $i$ & medium & \\
\hline K01115.01 & 13.739 & 2012 Aug 5 & $i$ & low & \\
\hline K01116.01 & 13.153 & 2012 Aug 5 & $i$ & medium & \\
\hline K01118.01 & 13.672 & 2012 Aug 5 & $i$ & medium & \\
\hline K01127.01 & 15.587 & 2012 Sep 3 & LP600 & low & \\
\hline K01128.01 & 13.277 & 2012 Aug 5 & $i$ & medium & \\
\hline K01141.01 & 15.39 & 2012 Aug 4 & LP600 & low & \\
\hline K01145.01 & 13.956 & 2012 Aug 5 & $i$ & low & \\
\hline K01146.01 & 15.043 & 2012 Jul 15 & LP600 & low & \\
\hline K01148.01 & 13.769 & 2012 Aug 5 & $i$ & medium & \\
\hline K01150.01 & 13.139 & 2012 Aug 5 & $i$ & medium & yes \\
\hline K01151.01 & 13.198 & 2012 Aug 5 & $i$ & medium & yes \\
\hline K01152.01 & 13.622 & 2012 Sep 14 & LP600 & low & yes \\
\hline K01161.01 & 14.391 & 2012 Sep 3 & LP600 & medium & \\
\hline K01162.01 & 12.622 & 2012 Aug 4 & $i$ & high & \\
\hline K01163.01 & 14.735 & 2012 Sep 3 & LP600 & medium & \\
\hline K01165.01 & 13.699 & 2012 Aug 5 & $i$ & medium & \\
\hline K01168.01 & 13.851 & 2012 Aug 5 & $i$ & low & \\
\hline K01169.01 & 13.071 & 2012 Aug 5 & $i$ & medium & \\
\hline K01175.01 & 13.075 & 2012 Aug 5 & $i$ & medium & \\
\hline K01194.01 & 15.391 & 2012 Sep 3 & LP600 & medium & \\
\hline K01198.01 & 15.165 & 2012 Sep 3 & LP600 & low & \\
\hline K01202.01 & 15.352 & 2012 Aug 4 & LP600 & low & \\
\hline K01203.01 & 15.159 & 2012 Sep 3 & LP600 & low & \\
\hline K01208.01 & 13.456 & 2012 Aug 6 & $i$ & medium & \\
\hline K01215.01 & 13.226 & 2012 Aug 6 & $i$ & medium & \\
\hline K01216.01 & 13.28 & 2012 Aug 5 & $i$ & low & \\
\hline K01218.01 & 13.13 & 2012 Aug 6 & $i$ & medium & \\
\hline K01220.01 & 12.713 & 2012 Aug 6 & $i$ & medium & \\
\hline K01221.01 & 11.265 & 2012 Aug 6 & $i$ & high & \\
\hline K01222.01 & 11.909 & 2012 Aug 6 & $i$ & high & \\
\hline K01227.01 & 13.785 & 2012 Sep 14 & LP600 & low & \\
\hline K01230.01 & 11.914 & 2012 Aug 6 & $i$ & high & \\
\hline K01236.01 & 13.518 & 2012 Aug 6 & $i$ & low & \\
\hline K01239.01 & 14.812 & 2012 Sep 3 & LP600 & medium & \\
\hline K01240.01 & 14.242 & 2012 Sep 3 & LP600 & medium & \\
\hline K01241.01 & 12.09 & 2012 Sep 14 & LP600 & high & \\
\hline K01242.01 & 13.611 & 2012 Aug 6 & $i$ & low & \\
\hline K01257.01 & 14.367 & 2012 Sep 14 & LP600 & low & \\
\hline K01258.01 & 15.528 & 2012 Sep 3 & LP600 & medium & \\
\hline K01266.01 & 14.869 & 2012 Jun 17 & LP600 & low & \\
\hline K01270.01 & 14.544 & 2012 Sep 3 & LP600 & medium & \\
\hline
\end{tabular}


Table 5

(Continued)

\begin{tabular}{|c|c|c|c|c|c|}
\hline KOI & $\mathrm{m}_{\mathrm{i}} / \mathrm{mags}$ & ObsID & Filter & Obs. qual. & Companion? \\
\hline K01271.01 & 13.5 & 2012 Aug 6 & $i$ & low & \\
\hline K01274.01 & 13.107 & 2012 Aug 6 & $i$ & medium & yes \\
\hline K01275.01 & 13.442 & 2012 Jul 28 & $i$ & low & \\
\hline K01276.01 & 14.542 & 2012 Sep 3 & LP600 & medium & \\
\hline K01278.01 & 15.02 & 2012 Sep 3 & LP600 & medium & \\
\hline K01279.01 & 13.555 & 2012 Aug 6 & $i$ & low & \\
\hline K01282.01 & 12.399 & 2012 Aug 6 & $i$ & high & \\
\hline K01283.01 & & 2012 Aug 6 & $i$ & high & \\
\hline K01288.01 & 14.967 & 2012 Sep 14 & LP600 & low & \\
\hline K01299.01 & 11.878 & 2012 Aug 6 & $i$ & high & \\
\hline K01301.01 & 15.581 & 2012 Sep 3 & LP600 & low & \\
\hline K01305.01 & 14.913 & 2012 Sep 3 & LP600 & medium & \\
\hline K01306.01 & 15.374 & 2012 Sep 4 & LP600 & low & \\
\hline K01307.01 & 14.551 & 2012 Sep 4 & LP600 & medium & \\
\hline K01308.01 & 13.781 & 2012 Aug 6 & $i$ & low & \\
\hline K01309.01 & 13.727 & 2012 Aug 6 & $i$ & medium & \\
\hline K01314.01 & 12.941 & 2012 Aug 6 & $i$ & medium & \\
\hline K01315.01 & 12.998 & 2012 Aug 6 & $i$ & medium & \\
\hline K01316.01 & 11.694 & 2012 Aug 6 & $i$ & high & \\
\hline K01332.01 & 14.919 & 2012 Sep 3 & LP600 & medium & \\
\hline K01335.01 & 13.774 & 2012 Aug 6 & $i$ & low & \\
\hline K01336.01 & 14.61 & 2012 Sep 4 & LP600 & medium & \\
\hline K01338.01 & 14.385 & $2012 \operatorname{Sep} 4$ & LP600 & medium & \\
\hline K01342.01 & 14.033 & 2012 Sep 4 & LP600 & medium & \\
\hline K01344.01 & 13.269 & 2012 Aug 6 & $i$ & medium & \\
\hline K01353.01 & 13.764 & 2012 Aug 6 & $i$ & medium & \\
\hline K01358.01 & 15.117 & 2012 Sep 4 & LP600 & low & \\
\hline K01359.01 & 15.025 & 2012 Sep 4 & LP600 & medium & yes \\
\hline K01360.01 & 15.293 & 2012 Sep 4 & LP600 & low & \\
\hline K01363.01 & 15.719 & 2012 Sep 4 & LP600 & low & \\
\hline K01364.01 & 15.669 & 2012 Sep 4 & LP600 & low & \\
\hline K01366.01 & 15.138 & 2012 Sep 4 & LP600 & medium & \\
\hline K01375.01 & 13.533 & 2012 Aug 6 & $i$ & medium & yes \\
\hline K01376.01 & 13.902 & 2012 Aug 6 & $i$ & medium & \\
\hline K01378.01 & 13.327 & 2012 Aug 6 & $i$ & medium & \\
\hline K01379.01 & 13.499 & 2012 Aug 6 & $i$ & medium & \\
\hline K01393.01 & 15.201 & 2012 Jul 15 & LP600 & low & \\
\hline K01396.01 & 15.62 & 2012 Sep 4 & LP600 & medium & \\
\hline K01401.01 & 13.316 & 2012 Aug 6 & $i$ & medium & \\
\hline K01408.01 & 14.141 & 2012 Aug 4 & LP600 & medium & \\
\hline K01412.01 & 13.434 & 2012 Aug 6 & $i$ & medium & \\
\hline K01422.01 & 15.194 & 2012 Aug 4 & LP600 & low & \\
\hline K01426.01 & 14.063 & 2012 Aug 6 & $i$ & medium & \\
\hline K01427.01 & 15.287 & 2012 Aug 4 & LP600 & medium & \\
\hline K01435.01 & 14.012 & 2012 Sep 4 & LP600 & medium & \\
\hline K01436.01 & 14.061 & 2012 Sep 4 & LP600 & medium & \\
\hline K01438.01 & 13.858 & 2012 Aug 6 & $i$ & medium & \\
\hline K01439.01 & 12.689 & 2012 Aug 6 & $i$ & medium & \\
\hline K01442.01 & 12.296 & 2012 Aug 6 & $i$ & high & yes \\
\hline K01444.01 & 13.784 & 2012 Aug 6 & $i$ & low & \\
\hline K01452.01 & 13.525 & 2012 Aug 6 & $i$ & medium & \\
\hline K01459.01 & 15.139 & 2012 Aug 4 & LP600 & medium & \\
\hline K01478.01 & 12.254 & 2012 Aug 6 & $i$ & high & \\
\hline K01480.01 & 15.573 & 2012 Sep 4 & LP600 & low & \\
\hline K01486.01 & 15.286 & 2012 Sep 4 & LP600 & medium & \\
\hline K01515.01 & 13.862 & 2012 Sep 4 & LP600 & medium & \\
\hline K01525.01 & 12.009 & 2012 Aug 6 & $i$ & high & \\
\hline K01528.01 & 13.822 & 2012 Aug 6 & $i$ & medium & \\
\hline K01529.01 & 14.152 & 2012 Sep 4 & LP600 & high & \\
\hline K01530.01 & 12.88 & 2012 Aug 6 & $i$ & medium & \\
\hline K01535.01 & 12.884 & 2012 Aug 6 & $i$ & medium & \\
\hline K01536.01 & 12.542 & 2012 Aug 6 & $i$ & medium & \\
\hline K01537.01 & & 2012 Aug 29 & $i$ & high & \\
\hline K01557.01 & 14.457 & 2012 Sep 4 & LP600 & medium & \\
\hline K01563.01 & 15.475 & 2012 Sep 4 & LP600 & low & \\
\hline
\end{tabular}

Table 5

(Continued)

\begin{tabular}{|c|c|c|c|c|c|}
\hline KOI & $\mathrm{m}_{\mathrm{i}} / \mathrm{mags}$ & ObsID & Filter & Obs. qual. & $\overline{\text { Companion? }}$ \\
\hline K01567.01 & 15.254 & 2012 Sep 4 & LP600 & medium & \\
\hline K01576.01 & 13.826 & 2012 Aug 6 & $i$ & medium & \\
\hline K01588.01 & 14.184 & 2012 Jun 17 & LP600 & medium & \\
\hline K01589.01 & 14.547 & 2012 Sep 4 & LP600 & medium & \\
\hline K01590.01 & 15.326 & 2012 Sep 4 & LP600 & low & \\
\hline K01596.01 & 14.758 & 2012 Jun 17 & LP600 & low & \\
\hline K01597.01 & 12.598 & 2012 Aug 6 & $i$ & high & \\
\hline K01598.01 & 14.063 & 2012 Sep 4 & LP600 & medium & \\
\hline K01606.01 & 13.752 & 2012 Aug 6 & $i$ & medium & \\
\hline K01608.01 & 13.647 & 2012 Sep 4 & LP600 & high & \\
\hline K01609.01 & 13.793 & 2012 Aug 29 & $i$ & low & \\
\hline K01612.01 & 8.658 & 2012 Aug 6 & $i$ & high & \\
\hline K01613.01 & & 2012 Aug 29 & $i$ & high & yes \\
\hline K01615.01 & 11.341 & 2012 Aug 29 & $i$ & high & \\
\hline K01616.01 & 11.396 & 2012 Aug 29 & $i$ & high & \\
\hline K01618.01 & 11.473 & 2012 Aug 29 & $i$ & high & \\
\hline K01619.01 & 11.427 & 2012 Aug 29 & $i$ & high & yes \\
\hline K01621.01 & 11.711 & 2012 Aug 29 & $i$ & high & \\
\hline K01622.01 & 12.033 & 2012 Aug 29 & $i$ & high & \\
\hline K01627.01 & 15.493 & 2012 Sep 4 & LP600 & low & \\
\hline K01628.01 & 12.775 & 2012 Aug 29 & $i$ & medium & \\
\hline K01629.01 & 13.381 & 2012 Aug 29 & $i$ & medium & \\
\hline K01632.01 & 13.157 & 2012 Aug 29 & $i$ & medium & \\
\hline K01647.01 & 13.961 & 2012 Sep 4 & LP600 & high & \\
\hline K01649.01 & 14.347 & 2012 Jul 16 & LP600 & medium & \\
\hline K01655.01 & 13.559 & 2012 Sep 14 & LP600 & medium & \\
\hline K01665.01 & 13.871 & 2012 Sep 4 & LP600 & high & \\
\hline K01669.01 & 14.018 & 2012 Sep 14 & LP600 & low & \\
\hline K01677.01 & 14.073 & 2012 Sep 4 & LP600 & medium & yes \\
\hline K01684.01 & 12.717 & 2012 Sep 14 & LP600 & high & \\
\hline K01692.01 & 12.313 & 2012 Sep 4 & LP600 & high & \\
\hline K01701.01 & 11.047 & 2012 Aug 4 & $i$ & high & \\
\hline K01706.01 & 13.835 & 2012 Sep 14 & LP600 & medium & \\
\hline K01713.01 & 14.712 & 2012 Sep 13 & LP600 & medium & \\
\hline K01715.01 & 12.751 & 2012 Aug 29 & $i$ & medium & \\
\hline K01725.01 & 13.107 & 2012 Aug 29 & $i$ & medium & \\
\hline K01726.01 & 12.684 & 2012 Aug 29 & $i$ & medium & \\
\hline K01738.01 & 13.032 & 2012 Aug 29 & $i$ & medium & \\
\hline K01751.01 & 14.248 & 2012 Sep 13 & LP600 & medium & \\
\hline K01754.01 & 13.775 & 2012 Sep 14 & LP600 & medium & \\
\hline K01779.01 & 13.077 & 2012 Aug 29 & $i$ & low & \\
\hline K01781.01 & 11.884 & 2012 Sep 13 & LP600 & high & \\
\hline K01783.01 & 13.774 & 2012 Sep 14 & LP600 & medium & \\
\hline K01802.01 & 13.175 & 2012 Aug 29 & $i$ & medium & \\
\hline K01803.01 & 12.932 & 2012 Aug 29 & $i$ & medium & \\
\hline K01805.01 & 13.591 & 2012 Sep 14 & LP600 & medium & \\
\hline K01812.01 & 13.582 & 2012 Aug 29 & $i$ & medium & \\
\hline K01813.01 & 13.525 & 2012 Aug 29 & $i$ & medium & \\
\hline K01814.01 & 12.453 & 2012 Sep 14 & LP600 & high & \\
\hline K01818.01 & 13.881 & 2012 Sep 14 & LP600 & medium & \\
\hline K01819.01 & 13.347 & 2012 Aug 29 & $i$ & medium & \\
\hline K01820.01 & 13.292 & 2012 Sep 13 & LP600 & high & \\
\hline K01822.01 & 12.281 & 2012 Aug 29 & $i$ & medium & \\
\hline K01824.01 & 12.567 & 2012 Aug 29 & $i$ & medium & \\
\hline K01825.01 & 13.632 & 2012 Sep 14 & LP600 & high & \\
\hline K01831.01 & 13.866 & 2012 Sep 14 & LP600 & medium & \\
\hline K01832.01 & 14.776 & 2012 Sep 13 & LP600 & low & \\
\hline K01835.01 & 13.388 & 2012 Sep 13 & LP600 & high & \\
\hline K01839.01 & 12.992 & 2012 Aug 29 & $i$ & medium & \\
\hline K01843.01 & 13.708 & 2012 Aug 29 & $i$ & medium & \\
\hline K01845.01 & 14.05 & 2012 Sep 13 & LP600 & medium & yes \\
\hline K01850.01 & 13.952 & 2012 Sep 14 & LP600 & medium & \\
\hline K01852.01 & 12.97 & 2012 Aug 29 & $i$ & medium & \\
\hline K01854.01 & 13.293 & 2012 Aug 29 & $i$ & medium & \\
\hline K01856.01 & 13.804 & 2012 Sep 14 & LP600 & medium & \\
\hline
\end{tabular}


Table 5

(Continued)

\begin{tabular}{|c|c|c|c|c|c|}
\hline KOI & $\mathrm{m}_{\mathrm{i}} / \mathrm{mags}$ & ObsID & Filter & Obs. qual. & Companion? \\
\hline K01857.01 & 13.548 & 2012 Aug 29 & $i$ & medium & \\
\hline K01860.01 & 13.822 & 2012 Sep 14 & LP600 & medium & \\
\hline K01862.01 & 13.453 & 2012 Aug 29 & $i$ & medium & \\
\hline K01863.01 & 13.473 & 2012 Aug 29 & $i$ & low & \\
\hline K01867.01 & 14.404 & 2012 Jul 15 & LP600 & low & \\
\hline K01868.01 & 14.652 & 2012 Jul 15 & LP600 & low & \\
\hline K01874.01 & 14.947 & 2012 Sep 13 & LP600 & low & \\
\hline K01878.01 & 12.835 & 2012 Aug 29 & $i$ & medium & \\
\hline K01880.01 & 13.835 & 2012 Jul 15 & LP600 & medium & yes \\
\hline K01883.01 & 11.757 & 2012 Aug 29 & $i$ & high & \\
\hline K01884.01 & 15.158 & 2012 Sep 13 & LP600 & low & yes \\
\hline K01886.01 & 12.087 & 2012 Aug 29 & $i$ & high & \\
\hline K01888.01 & 13.15 & 2012 Aug 29 & $i$ & medium & \\
\hline K01889.01 & 15.109 & 2012 Sep 13 & LP600 & medium & \\
\hline K01890.01 & 11.555 & 2012 Aug 29 & $i$ & high & yes \\
\hline K01891.01 & 14.957 & 2012 Sep 13 & LP600 & medium & yes \\
\hline K01893.01 & 13.876 & 2012 Sep 14 & LP600 & medium & \\
\hline K01894.01 & 13.05 & 2012 Sep 14 & LP600 & high & \\
\hline K01895.01 & 15.42 & 2012 Sep 13 & LP600 & low & \\
\hline K01897.01 & 13.779 & 2012 Sep 14 & LP600 & high & \\
\hline K01905.01 & 13.713 & 2012 Sep 14 & LP600 & medium & \\
\hline K01907.01 & 14.699 & 2012 Jul 15 & LP600 & low & \\
\hline K01909.01 & 12.612 & 2012 Sep 13 & LP600 & high & \\
\hline K01913.01 & 13.083 & 2012 Aug 29 & $i$ & medium & \\
\hline K01915.01 & 13.809 & 2012 Sep 14 & LP600 & medium & \\
\hline K01916.01 & 13.42 & 2012 Sep 13 & LP600 & high & yes \\
\hline K01917.01 & 13.479 & 2012 Aug 29 & $i$ & medium & \\
\hline K01921.01 & 12.708 & 2012 Sep 14 & LP600 & high & \\
\hline K01922.01 & 15.159 & 2012 Sep 13 & LP600 & medium & \\
\hline K01923.01 & 13.879 & 2012 Aug 29 & $i$ & low & \\
\hline K01924.01 & 7.674 & 2012 Aug 29 & $i$ & high & \\
\hline K01925.01 & 9.211 & 2012 Aug 29 & $i$ & high & \\
\hline K01929.01 & 12.53 & 2012 Sep 13 & LP600 & high & \\
\hline K01930.01 & 11.957 & 2012 Sep 13 & LP600 & high & \\
\hline K01931.01 & 14.307 & 2012 Sep 13 & LP600 & medium & \\
\hline K01932.01 & 12.366 & 2012 Sep 14 & LP600 & high & \\
\hline K01938.01 & 13.766 & 2012 Sep 14 & LP600 & medium & \\
\hline K01940.01 & 14.912 & 2012 Sep 13 & LP600 & medium & \\
\hline K01944.01 & 13.79 & 2012 Sep 14 & LP600 & medium & \\
\hline K01945.01 & 14.267 & 2012 Sep 13 & LP600 & medium & \\
\hline K01952.01 & 14.398 & 2012 Sep 13 & LP600 & medium & \\
\hline K01955.01 & 13.025 & 2012 Sep 13 & LP600 & high & \\
\hline K01960.01 & 13.975 & 2012 Sep 14 & LP600 & low & \\
\hline K01961.01 & 12.61 & 2012 Aug 30 & $i$ & medium & \\
\hline K01962.01 & & 2012 Aug 30 & $i$ & high & yes \\
\hline K01964.01 & 10.464 & 2012 Aug 30 & $i$ & high & yes \\
\hline K01970.01 & 15.141 & 2012 Sep 13 & LP600 & low & \\
\hline K01977.01 & 13.566 & 2012 Oct 6 & LP600 & high & \\
\hline K01979.01 & 12.786 & 2012 Aug 30 & $i$ & medium & yes \\
\hline K01984.01 & 13.528 & 2012 Aug 30 & $i$ & medium & \\
\hline K01988.01 & 13.741 & 2012 Sep 14 & LP600 & medium & \\
\hline K02001.01 & 12.82 & 2012 Aug 30 & $i$ & medium & \\
\hline K02002.01 & 13.104 & 2012 Aug 30 & $i$ & medium & \\
\hline K02004.01 & 13.15 & 2012 Aug 30 & $i$ & medium & \\
\hline K02006.01 & 13.626 & 2012 Jul 16 & LP600 & high & \\
\hline K02009.01 & 13.616 & 2012 Sep 14 & LP600 & medium & yes \\
\hline K02010.01 & 13.054 & 2012 Aug 30 & $i$ & medium & \\
\hline K02011.01 & 12.419 & 2012 Sep 14 & LP600 & high & \\
\hline K02013.01 & 12.665 & 2012 Aug 30 & $i$ & medium & \\
\hline K02016.01 & 13.954 & 2012 Sep 14 & LP600 & medium & \\
\hline K02017.01 & 12.888 & 2012 Aug 30 & $i$ & medium & \\
\hline K02022.01 & 14.551 & 2012 Sep 13 & LP600 & medium & \\
\hline K02025.01 & 13.608 & 2012 Sep 13 & LP600 & high & \\
\hline K02026.01 & 13.121 & 2012 Aug 30 & $i$ & medium & \\
\hline K02029.01 & 12.694 & 2012 Sep 13 & LP600 & high & \\
\hline
\end{tabular}

Table 5

(Continued)

\begin{tabular}{|c|c|c|c|c|c|}
\hline KOI & $\mathrm{m}_{\mathrm{i}} / \mathrm{mags}$ & ObsID & Filter & Obs. qual. & Companion? \\
\hline K02033.01 & 13.476 & 2012 Aug 30 & $i$ & medium & \\
\hline K02035.01 & 12.782 & 2012 Aug 31 & $i$ & medium & \\
\hline K02038.01 & 14.548 & 2012 Oct 6 & LP600 & medium & \\
\hline K02040.01 & 13.983 & 2012 Oct 6 & LP600 & high & \\
\hline K02042.01 & 12.941 & 2012 Aug 31 & $i$ & low & \\
\hline K02044.01 & 15.591 & 2012 Aug 30 & LP600 & low & \\
\hline K02045.01 & 15.135 & 2012 Sep 13 & LP600 & low & \\
\hline K02046.01 & 12.939 & 2012 Aug 30 & $i$ & medium & \\
\hline K02047.01 & 13.845 & 2012 Oct 6 & LP600 & high & \\
\hline K02049.01 & 13.771 & 2012 Oct 6 & LP600 & high & \\
\hline K02051.01 & 14.902 & 2012 Sep 13 & LP600 & low & \\
\hline K02053.01 & 12.839 & 2012 Sep 13 & LP600 & high & \\
\hline K02057.01 & 14.432 & 2012 Jul 16 & LP600 & medium & \\
\hline K02058.01 & 14.78 & 2012 Jul 16 & LP600 & low & \\
\hline K02059.01 & 12.558 & 2012 Oct 6 & LP600 & high & yes \\
\hline K02071.01 & 13.478 & 2012 Aug 30 & $i$ & medium & \\
\hline K02072.01 & 13.215 & 2012 Aug 30 & $i$ & medium & \\
\hline K02073.01 & 15.225 & 2012 Sep 13 & LP600 & medium & \\
\hline K02079.01 & 12.709 & 2012 Aug 30 & $i$ & medium & \\
\hline K02082.01 & 13.964 & 2012 Oct 6 & LP600 & high & \\
\hline K02086.01 & 13.776 & 2012 Oct 6 & LP600 & high & \\
\hline K02087.01 & 11.727 & 2012 Aug 30 & $i$ & high & \\
\hline K02090.01 & 14.88 & 2012 Jul 16 & LP600 & low & \\
\hline K02105.01 & 13.693 & 2012 Oct 6 & LP600 & high & \\
\hline K02110.01 & 12.071 & 2012 Aug 30 & $i$ & high & \\
\hline K02111.01 & 14.674 & 2012 Sep 13 & LP600 & low & \\
\hline K02119.01 & 13.799 & 2012 Oct 6 & LP600 & high & \\
\hline K02133.01 & 12.104 & 2012 Aug 31 & $i$ & medium & \\
\hline K02135.01 & 13.416 & 2012 Aug 30 & $i$ & medium & \\
\hline K02137.01 & 13.489 & 2012 Aug 30 & $i$ & medium & \\
\hline K02138.01 & 12.127 & 2012 Aug 30 & $i$ & high & \\
\hline K02143.01 & 13.872 & 2012 Oct 6 & LP600 & high & yes \\
\hline K02149.01 & 11.928 & 2012 Aug 30 & $i$ & high & \\
\hline K02158.01 & 12.796 & 2012 Jul 28 & $i$ & medium & \\
\hline K02159.01 & 13.293 & 2012 Aug 31 & $i$ & medium & yes \\
\hline K02162.01 & 13.864 & 2012 Oct 6 & LP600 & high & \\
\hline K02169.01 & 12.172 & 2012 Sep 13 & LP600 & high & \\
\hline K02173.01 & 12.522 & 2012 Sep 13 & LP600 & high & \\
\hline K02175.01 & 12.626 & 2012 Oct 6 & LP600 & high & \\
\hline K02191.01 & 14.275 & 2012 Jul 17 & LP600 & medium & \\
\hline K02194.01 & 13.681 & 2012 Aug 31 & $i$ & low & \\
\hline K02201.01 & 13.618 & 2012 Oct 6 & LP600 & high & \\
\hline K02202.01 & 13.842 & 2012 Aug 31 & $i$ & low & \\
\hline K02204.01 & 13.8 & 2012 Oct 6 & LP600 & medium & \\
\hline K02215.01 & 12.699 & 2012 Aug 31 & $i$ & medium & \\
\hline K02219.01 & 13.781 & 2012 Aug 31 & $i$ & low & \\
\hline K02220.01 & 14.48 & 2012 Sep 13 & LP600 & low & \\
\hline K02222.01 & 12.875 & 2012 Aug 31 & $i$ & medium & \\
\hline K02224.01 & 14.742 & 2012 Sep 13 & LP600 & medium & \\
\hline K02228.01 & 12.61 & 2012 Oct 6 & LP600 & high & \\
\hline K02238.01 & 14.037 & 2012 Jul 17 & LP600 & medium & \\
\hline K02246.01 & 13.965 & 2012 Aug 31 & $i$ & low & \\
\hline K02252.01 & 13.471 & 2012 Aug 31 & $i$ & medium & \\
\hline K02260.01 & 12.05 & 2012 Aug 31 & $i$ & high & \\
\hline K02272.01 & 12.747 & 2012 Aug 31 & $i$ & high & \\
\hline K02273.01 & 12.553 & 2012 Aug 31 & $i$ & medium & \\
\hline K02276.01 & 11.485 & 2012 Aug 31 & $i$ & high & \\
\hline K02279.01 & 13.688 & 2012 Oct 6 & LP600 & high & \\
\hline K02281.01 & 13.535 & 2012 Oct 6 & LP600 & high & \\
\hline K02287.01 & 12.1 & 2012 Aug 31 & $i$ & high & \\
\hline K02289.01 & 13.193 & 2012 Aug 31 & $i$ & medium & \\
\hline K02300.01 & 13.799 & 2012 Aug 31 & $i$ & low & \\
\hline K02303.01 & 13.71 & 2012 Oct 6 & LP600 & high & \\
\hline K02312.01 & 12.586 & 2012 Aug 31 & $i$ & high & \\
\hline K02319.01 & 13.224 & 2012 Aug 31 & $i$ & medium & \\
\hline
\end{tabular}


Table 5

(Continued)

\begin{tabular}{|c|c|c|c|c|c|}
\hline KOI & $\mathrm{m}_{\mathrm{i}} / \mathrm{mags}$ & ObsID & Filter & Obs. qual. & Companion? \\
\hline K02331.01 & 13.29 & 2012 Aug 31 & $i$ & medium & \\
\hline K02332.01 & 12.766 & 2012 Aug 31 & $i$ & medium & \\
\hline K02335.01 & 13.912 & 2012 Oct 6 & LP600 & high & \\
\hline K02342.01 & 12.87 & 2012 Aug 31 & $i$ & medium & \\
\hline K02347.01 & 14.369 & 2012 Jul 17 & LP600 & low & \\
\hline K02352.01 & & 2012 Sep 14 & LP600 & high & \\
\hline K02358.01 & 13.383 & 2012 Aug 31 & $i$ & medium & \\
\hline K02365.01 & 13.682 & 2012 Oct 6 & LP600 & medium & \\
\hline K02366.01 & 12.337 & 2012 Aug 31 & $i$ & high & \\
\hline K02367.01 & 12.475 & 2012 Oct 6 & LP600 & high & \\
\hline K02370.01 & 12.878 & 2012 Jul 28 & $i$ & medium & \\
\hline K02374.01 & 14.371 & 2012 Sep 14 & LP600 & low & \\
\hline K02389.01 & 13.417 & 2012 Aug 31 & $i$ & low & \\
\hline K02390.01 & 12.08 & 2012 Aug 31 & $i$ & high & \\
\hline K02398.01 & 13.437 & 2012 Aug 31 & $i$ & medium & \\
\hline K02399.01 & 13.833 & 2012 Oct 6 & LP600 & medium & \\
\hline K02407.01 & 13.979 & 2012 Aug 31 & $i$ & low & \\
\hline K02408.01 & 13.972 & 2012 Aug 31 & $i$ & low & \\
\hline K02410.01 & 14.949 & 2012 Sep 14 & LP600 & low & \\
\hline K02413.01 & 14.684 & 2012 Sep 14 & LP600 & low & yes \\
\hline K02414.01 & 13.39 & 2012 Sep 14 & LP600 & medium & \\
\hline K02426.01 & 13.658 & 2012 Oct 6 & LP600 & high & \\
\hline K02433.01 & 15.041 & 2012 Sep 14 & LP600 & low & \\
\hline K02440.01 & 13.762 & 2012 Oct 6 & LP600 & high & \\
\hline K02443.01 & 13.83 & 2012 Oct 6 & LP600 & high & yes \\
\hline K02457.01 & 12.267 & 2012 Aug 31 & $i$ & medium & \\
\hline K02463.01 & 12.609 & 2012 Aug 31 & $i$ & medium & yes \\
\hline K02470.01 & 13.448 & 2012 Aug 31 & $i$ & medium & \\
\hline K02479.01 & 12.687 & 2012 Oct 6 & LP600 & high & \\
\hline K02481.01 & 13.214 & 2012 Aug 31 & $i$ & medium & \\
\hline K02484.01 & 12.293 & 2012 Aug 31 & $i$ & high & \\
\hline K02486.01 & 12.89 & 2012 Aug 31 & $i$ & medium & yes \\
\hline K02488.01 & 13.395 & 2012 Aug 31 & $i$ & medium & \\
\hline K02498.01 & 13.678 & 2012 Oct 6 & LP600 & high & \\
\hline K02503.01 & 13.781 & 2012 Aug 31 & $i$ & medium & \\
\hline $\mathrm{K} 02522.01$ & 13.356 & 2012 Aug 31 & $i$ & medium & \\
\hline K02527.01 & 13.67 & 2012 Oct 6 & LP600 & high & \\
\hline K02530.01 & 13.436 & 2012 Aug 31 & $i$ & medium & \\
\hline K02533.01 & 12.967 & 2012 Aug 31 & $i$ & medium & \\
\hline K02534.01 & 13.755 & 2012 Oct 6 & LP600 & high & \\
\hline K02538.01 & 13.847 & 2012 Oct 6 & LP600 & high & \\
\hline K02541.01 & 12.717 & 2012 Aug 31 & $i$ & medium & \\
\hline K02545.01 & 11.63 & 2012 Aug 31 & $i$ & high & \\
\hline K02547.01 & 13.976 & 2012 Oct 6 & LP600 & high & \\
\hline K02555.01 & 12.756 & 2012 Oct 6 & LP600 & high & \\
\hline K02556.01 & 13.828 & 2012 Oct 6 & LP600 & medium & \\
\hline K02559.01 & 13.626 & 2012 Aug 31 & $i$ & medium & \\
\hline K02561.01 & 13.49 & 2012 Aug 31 & $i$ & medium & \\
\hline K02563.01 & 13.82 & 2012 Oct 6 & LP600 & high & \\
\hline K02564.01 & 13.91 & 2012 Oct 6 & LP600 & high & \\
\hline K02581.01 & 13.248 & 2012 Aug 31 & $i$ & medium & \\
\hline K02582.01 & 13.45 & 2012 Aug 31 & $i$ & medium & \\
\hline K02583.01 & 12.423 & 2012 Oct 6 & LP600 & high & \\
\hline K02585.01 & 13.311 & 2012 Aug 31 & $i$ & medium & \\
\hline K02593.01 & & 2012 Aug 31 & $i$ & high & \\
\hline K02595.01 & 13.107 & 2012 Aug 31 & $i$ & medium & \\
\hline K02597.01 & 14.626 & 2012 Sep 14 & LP600 & low & \\
\hline K02603.01 & 12.457 & 2012 Oct 6 & LP600 & high & \\
\hline K02608.01 & 13.124 & 2012 Aug 31 & $i$ & medium & \\
\hline K02631.01 & 13.295 & 2012 Aug 31 & $i$ & medium & \\
\hline K02632.01 & 11.28 & 2012 Aug 31 & $i$ & high & \\
\hline K02640.01 & 12.896 & 2012 Aug 31 & $i$ & medium & \\
\hline K02641.01 & 13.63 & 2012 Oct 6 & LP600 & high & yes \\
\hline K02657.01 & 12.655 & 2012 Oct 6 & LP600 & high & yes \\
\hline K02662.01 & 13.739 & 2012 Jul 17 & LP600 & medium & \\
\hline
\end{tabular}

particularly S. Kunsman, M. Doyle, J. Henning, R. Walters, G. Van Idsinga, B. Baker, K. Dunscombe and D. Roderick. We recognize and acknowledge the very significant cultural role and reverence that the summit of Mauna Kea has always had within the indigenous Hawaiian community. We are most fortunate to have the opportunity to conduct observations from this mountain. C.B. and J.A.J. acknowledge support from the Alfred P. Sloan Foundation. J.A.J. acknowledges support from the David and Lucile Packard Foundation.

Facilities: PO:1.5m (Robo-AO), Keck:II (NIRC2-NGS)

\section{APPENDIX}

In Table 5, we list our Robo-AO observed KOIs, including the date the target was observed, the filter, the observation quality, and the presence of detected companions.

\section{REFERENCES}

Adams, E. R., Ciardi, D. R., Dupree, A. K., et al. 2012, AJ, 144, 42

Adams, E. R., Dupree, A. K., Kulesa, C., \& McCarthy, D. 2013, AJ, 146, 9 Baranec, C., Riddle, R., Law, N. M., et al. 2013, J. Visualized Exp., 72, e50021 Baranec, C., Riddle, R., Ramaprakash, A. N., et al. 2012, Proc. SPIE, 8447, 844704

Barclay, T., Rowe, J. F., Lissauer, J. J., et al. 2013, Natur, 494, 452

Barrado, D., Lillo-Box, J., Bouy, H., Aceituno, J., \& Sánchez, S. 2013, in European Physical Journal Web of Conferences, Vol. 47, European Physical Journal Web of Conferences, 5008

Batalha, N. M., Rowe, J. F., Bryson, S. T., et al. 2013, ApJS, 204, 24

Batygin, K. 2012, Natur, 491, 418

Borucki, W. J., Koch, D., Basri, G., et al. 2010, Sci, 327, 977

Borucki, W. J., Koch, D. G., Basri, G., et al. 2011, ApJ, 736, 19

Brown, T. M., Latham, D. W., Everett, M. E., \& Esquerdo, G. A. 2011, AJ, 142,112

Bryson, S. T., Jenkins, J. M., Gilliland, R. L., et al. 2013, PASP, 125, 889

Buchhave, L. A., Latham, D. W., Carter, J. A., et al. 2011, ApJS, 197, 3

Burgasser, A. J., Kirkpatrick, J. D., Reid, I. N., et al. 2003, ApJ, 586, 512

Cenko, S. B., Fox, D. B., Moon, D.-S., et al. 2006, PASP, 118, 1396

Colón, K. D., Ford, E. B., \& Morehead, R. C. 2012, MNRAS, 426, 342

Daemgen, S., Hormuth, F., Brandner, W., et al. 2009, A\&A, 498, 567

Dotter, A., Chaboyer, B., Jevremović, D., et al. 2008, ApJS, 178, 89

Dressing, C. D., \& Charbonneau, D. 2013, ApJ, 767, 95

Fabrycky, D., \& Tremaine, S. 2007, ApJ, 669, 1298

Feigelson, E. D., \& Jogesh Babu, G. 2012, Modern Statistical Methods for Astronomy (Cambridge: Cambridge Univ. Press)

Fressin, F., Torres, G., Charbonneau, D., et al. 2013, ApJ, 766, 81

Horch, E. P., Howell, S. B., Everett, M. E., \& Ciardi, D. R. 2012, AJ, 144,165

Howell, S. B., Everett, M. E., Sherry, W., Horch, E., \& Ciardi, D. R. 2011, AJ, 142,19

Johnson, J. A., Apps, K., Gazak, J. Z., et al. 2011, ApJ, 730, 79

Katz, B., Dong, S., \& Malhotra, R. 2011, PhRvL, 107, 181101

Koch, D. G., Borucki, W. J., Basri, G., et al. 2010, ApJL, 713, L79

Lafrenière, D., Marois, C., Doyon, R., Nadeau, D., \& Artigau, É. 2007, ApJ, 660,770

Law, N. M., Hodgkin, S. T., \& Mackay, C. D. 2006b, MNRAS, 368, 1917

Law, N. M., Kraus, A. L., Street, R., et al. 2012, ApJ, 757, 133

Law, N. M., Mackay, C. D., \& Baldwin, J. E. 2006a, A\&A, 446, 739

Law, N. M., Mackay, C. D., Dekany, R. G., et al. 2009, ApJ, 692, 924

Lillo-Box, J., Barrado, D., \& Bouy, H. 2012, A\&A, 546, A10

Marcy, G. W., Isaacson, H., Howard, A. W., et al. 2014, ApJS, 210, 20

Ming, Y., Hui-Gen, L., Hui, Z., Jia-Yi, Y., \& Ji-Lin, Z. 2013, ApJ, 778, 110

Morton, T. D. 2012, ApJ, 761, 6

Morton, T. D., \& Johnson, J. A. 2011, ApJ, 738, 170

Naoz, S., Farr, W. M., \& Rasio, F. A. 2012, ApJL, 754, L36

O’Donovan, F. T., Charbonneau, D., Torres, G., et al. 2006, ApJ, 644, 1237

Riddle, R. L., Burse, M. P., Law, N. M., et al. 2012, Proc. SPIE, 8447, $84472 \mathrm{O}$

Santerne, A., Fressin, F., Díaz, R. F., et al. 2013, A\&A, 557, A139

Tenenbaum, P., Jenkins, J. M., Seader, S., et al. 2013, ApJS, 206, 5

Terziev, E., Law, N. M., Arcavi, I., et al. 2013, ApJS, 206, 18

Wizinowich, P., Acton, D. S., Shelton, C., et al. 2000, PASP, 112, 315

York, D. G., Adelman, J., Anderson, J. E., Jr., et al. 2000, AJ, 120, 1579 